\title{
Mixing in turbulent jets: scalar measures and isosurface geometry
}

\author{
By HARIS J. CATRAKIS AND PAUL E. DIMOTAKIS \\ Graduate Aeronautical Laboratories, California Institute of Technology, \\ Pasadena, CA 91125, USA
}

(Received 5 July 1995 and in revised form 21 December 1995)

\begin{abstract}
Experiments have been conducted to investigate mixing and the geometry of scalar isosurfaces in turbulent jets. Specifically, we have obtained high-resolution, highsignal-to-noise-ratio images of the jet-fluid concentration in the far field of round, liquid-phase, turbulent jets, in the Reynolds number range $4.5 \times 10^{3} \leqslant R e \leqslant 18 \times 10^{3}$, using laser-induced-fluorescence imaging techniques. Analysis of these data indicates that this Reynolds-number range spans a mixing transition in the far field of turbulent jets. This is manifested in the probability-density function of the scalar field, as well as in measures of the scalar isosurfaces. Classical as well as fractal measures of these isosurfaces have been computed, from small to large spatial scales, and are found to be functions of both scalar threshold and Reynolds number. The coverage of level sets of jet-fluid concentration in the two-dimensional images is found to possess a scale-dependent-fractal dimension that increases continuously with increasing scale, from near unity, at the smallest scales, to 2 , at the largest scales. The geometry of the scalar isosurfaces is, therefore, more complex than power-law fractal, exhibiting an increasing complexity with increasing scale. This behaviour necessitates a scaledependent generalization of power-law-fractal geometry. A connection between scaledependent-fractal geometry and the distribution of scales is established and used to compute the distribution of spatial scales in the flow.
\end{abstract}

\section{Introduction}

In the turbulent mixing of a passive scalar, surfaces of constant mixed-fluid concentration, or isoscalar surfaces, are highly convoluted in turbulent-jet flows (è.g. Dimotakis, Miake-Lye \& Papantoniou 1983), as well as other high-Reynolds-number turbulent flows (e.g. Sreenivasan \& Meneveau 1986). Knowledge of the geometry of these isosurfaces is necessary for an understanding of the turbulent mixing process. In particular, molecular mixing occurs across such surfaces. In the case of non-premixed hydrocarbon combustion, for example, chemical reactions are largely confined to the instantaneous (isoscalar) stoichiometric surface (e.g. Bilger 1980), while in the case of premixed combustion, burning takes place on the (isotemperature) interface between burnt and unburnt fuel (e.g. Mantzaras 1992), with flame-front propagation characteristics that are intimately intertwined with the geometric complexity of the burning surface (e.g. Ashurst 1995). Various measures of the isosurface geometry, such as surface-to-volume ratio or volume-fill fraction, and their relation to the distribution of spatial scales, are, as a consequence, important to our understanding and modelling of mixing, chemical reactions, and combustion in turbulent flows.

Characterizations of the geometry of isosurfaces in turbulence, in particular, and 
of other complex structures encountered in natural phenomena, in general, require extending notions of Euclidean geometry. One such extension was proposed by Mandelbrot $(1967,1975 b, 1977,1982)$ who suggested that fractional (non-integer) dimensions can be used to describe complex geometries in nature. The word fractal was coined by Mandelbrot $(1975 a, b)$ to denote geometric objects that exhibit power-law scaling, i.e. objects characterized by constant, fractional (in general) dimensions. $\mathrm{He}$ regarded such objects as, "... rough and fragmented to the same degree at all scales" (Mandelbrot 1989). This power-law-fractal (denoted as 'PLF', below) geometry has been applied to the description of a wide variety of natural phenomena (e.g. Mandelbrot 1982). In these descriptions, PLFs are used to characterize the geometry in a finite range of scales that is bounded by upper- and lower-cutoff scales, "a property we can assume for all objects arising as a result of any physical process" (Vicsek 1992). The underlying point of view, in such descriptions of natural objects as PLFs, is that, "... over certain ranges of scale they appear very much like [power-law] fractals and at such scales may usefully be regarded as such" (Falconer 1990, p. xxi) - square-bracket insert ours. Fluid turbulence, a phenomenon long recognized to possess complex structure, was considered by Mandelbrot $(1975 a, b)$ as a candidate for PLF geometry.

In this work, we investigate mixing and the geometry of scalar isosurfaces in turbulence. In particular, the behaviour and properties of scalar level sets in turbulent jets, identified from two-dimensional image slices of the scalar field, are analysed using both classical and fractal measures. Section 2 describes the experimental procedure and imaging techniques. Section 3 analyses classical scalar and isoscalar measures, computed from the scalar-field images. Reynolds-number as well as scalar-threshold dependence is found for both classical and fractal measures. An account of PLF geometry, including reports of PLF behaviour in turbulent flows, is given in $\S 4$. Our findings necessitate an extension of the PLF framework to characterize the more complex geometries observed in these experiments, as well as in other natural phenomena, for which PLF descriptions cannot capture the observed behaviour. Section 5 describes the framework of scale-dependent-fractal (denoted as SDF, below) geometry, in which geometric behaviour is characterized by a fractal dimension that varies with scale, as opposed to scale-independent, PLF behaviour. It is proposed, in $\$ 5$, that turbulent flows, in particular, and complex natural phenomena, in general, may be expected to exhibit SDF behaviour, as opposed to PLF behaviour. Section 6 establishes a connection between fractal dimensions (SDF or PLF) and the distribution of geometric scales. Section 7 presents experimental evidence of SDF behaviour in turbulent-jet mixing, as well as its relation to the distribution of spatial scales in the flow.

\section{Experiments and imaging techniques}

We report on experiments in liquid-phase turbulent-jet flows in which images of slices through the three-dimensional scalar field of round momentum-driven turbulent jets were obtained. A schematic of the flow facility and imaging diagnostics is shown in figure 1. Transverse sections in the far field of the jet, at a downstream station $z / d_{0}=275$, where $d_{0}=2.54 \mathrm{~mm}$ is the (internal) jet-nozzle diameter, were recorded on a cryogenically cooled $(1024 \times 1024)$-pixel CCD camera (Photometrics Series 200 system), with a Nikon $50 \mathrm{~mm} \mathrm{f/1.2} \mathrm{lens,} \mathrm{using} \mathrm{planar} \mathrm{laser-induced} \mathrm{fluorescence}$ imaging techniques. A window at the bottom of the tank provided optical access with a $45^{\circ}$ mirror facilitating imaging from underneath the tank. The jet plenum was seeded with fluorescent dye (disodium fluorescein) at a concentration of $c_{0} \simeq 2.0 \times 10^{-6} \mathrm{M}$. The scalar-species Schmidt number is estimated to be $S c \simeq 1.9 \times 10^{3}$, based on the 


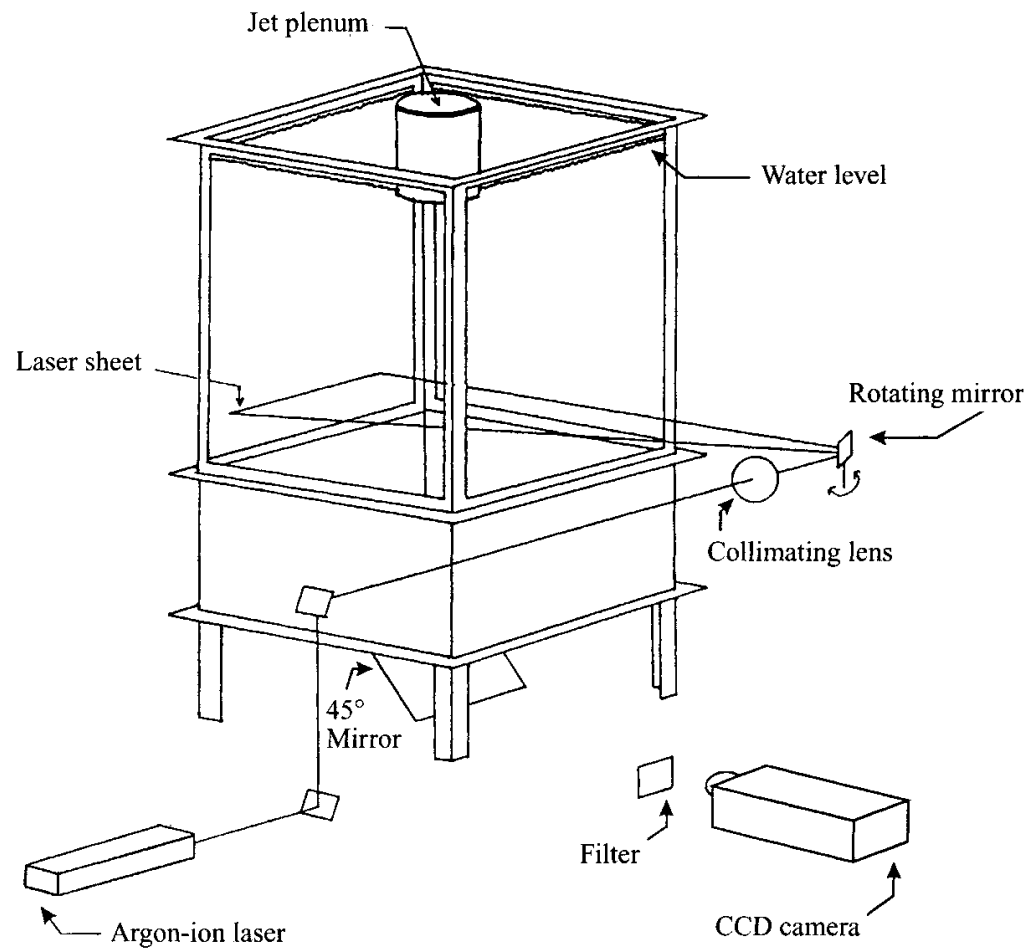

FIGURE 1. Flow facility and imaging schematic.

diffusion coefficient for disodium fluorescein, $\mathscr{D} \simeq 5.2 \times 10^{-6} \mathrm{~cm}^{2} \mathrm{~s}^{-1}$ (cf. Ware et al. 1983 , p. 280). At the downstream station used for these experiments $\left(z / d_{0}=275\right)$, we verified that the low plenum dye concentration resulted in a scalar fluorescent dye field with negligible laser attenuation across the field of view. The field of view spans $\ell_{0} \simeq 42 \mathrm{~cm}$, resulting in a pixel resolution of $\lambda_{\mathrm{p}} \simeq 420 \mu \mathrm{m}$, and contains the full transverse spatial extent of the turbulent-jet fluid at the measuring station. A Gaussian $\left(\mathrm{TEM}_{00}\right)$ beam from a continuous-wave Argon-ion laser (Coherent Innova 90) was collimated by a long-focal-length $(f=1000 \mathrm{~mm})$ spherical lens to a beam waist (diameter) of $w_{0} \lesssim 300 \mu \mathrm{m}$ and approximately positioned in the centre of the imaged field of view. The Rayleigh range for this beam was estimated to be $\pm 12.5 \mathrm{~cm}$ (on either side of the waist). This resulted in a transverse resolution that was smaller than the in-plane (pixel) resolution in the centre and comparable to it near the edges of the field of view. The laser beam was swept across the jet and synchronized with the CCD camera shutter using a small-inertia small-aperture $(5 \mathrm{~mm})$, galvanometrically driven linearly scanned mirror (General Scanning: Mirror M0540V and scanner G120DT). Background laser light was filtered out using a low-pass optical filter (Kodak No. 22). The experiments were conducted in a dark environment to minimize noise due to ambient light. More information on the facility, including the flow-management system, can be found in Miller \& Dimotakis $(1991 b)$ and references therein.

Figure 2 shows a schematic of the geometry of the scalar far field of the jet and the imaging station. The laser-illumination sheet was at constant $z / d_{0}$, i.e. in the similarity plane of the jet, and spanned the entire extent of the scalar field at that downstream location. The laser-induced-fluorescence image data were processed using a pixelby-pixel calibration of the CCD array for noise, sensitivity, and laser illumination variations in the field of view. Each raw fluorescence-data image, $I_{\text {raw }}(x, y)$, was 


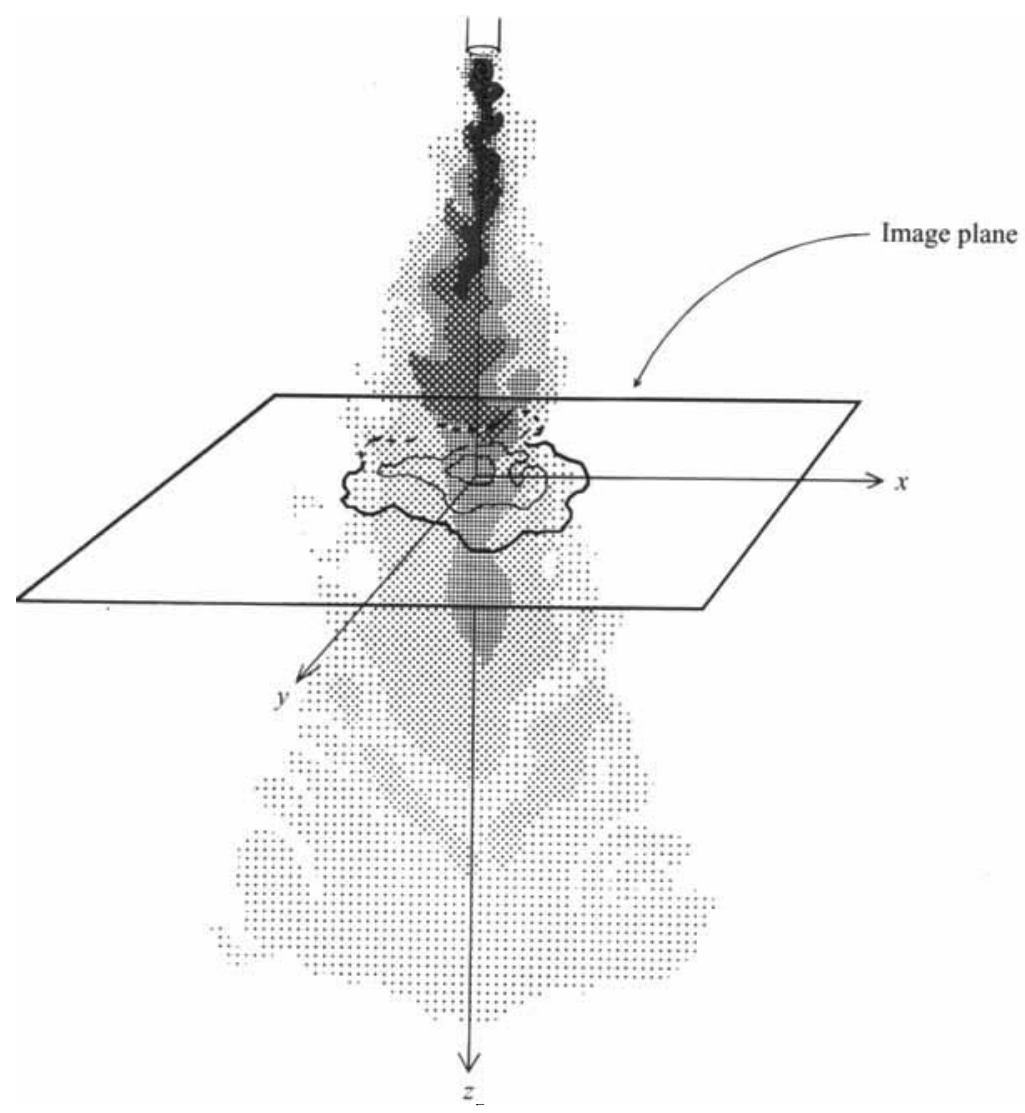

Figure 2. Jet scalar far field geometry and image plane schematic.

calibrated and normalized with an ensemble-average of four background-noise images and four uniform-concentration images recorded for each run condition, i.e.

$$
\frac{c(x, y ; z=\mathrm{const})}{c_{\text {ref }}}=\frac{I_{\text {raw }}(x, y)-\left\langle I_{b c k}(x, y)\right\rangle}{\left\langle I_{i l l}(x, y)\right\rangle-\left\langle I_{b c k}(x, y)\right\rangle},
$$

where $c_{\text {ref }}$ is a reference concentration that is constant for all runs. The backgroundnoise images, $I_{b c k}(x, y)$, were recorded shortly before each run with no dye in the tank. Each such image was recorded at the same location as the (raw) jet images, along the same optical path, with the camera shutter open for the same exposure time as for the jet images. After the jet-plenum fluid had completely discharged into the tank, all of the water and dye were recirculated continuously in the tank. During the recirculation process, the laser-induced fluorescence at the imaging station was monitored for nonuniformities of the dye concentration in the plane of illumination. After a sufficiently long time, non-uniformities were no longer measurable and images corresponding to the resulting uniform-concentration dye field were recorded and used to measure the illumination and optical collection efficiency distribution in the field of view, $I_{i l l}(x, y)$, as well as provide an absolute jet-fluid concentration reference. The procedure of emptying all the jet-plenum fluid into the tank ensured that $c_{r e f}$ was the same for all runs, and allowed the local scalar values, $c(x, y)$, to be referenced, in absolute value, 


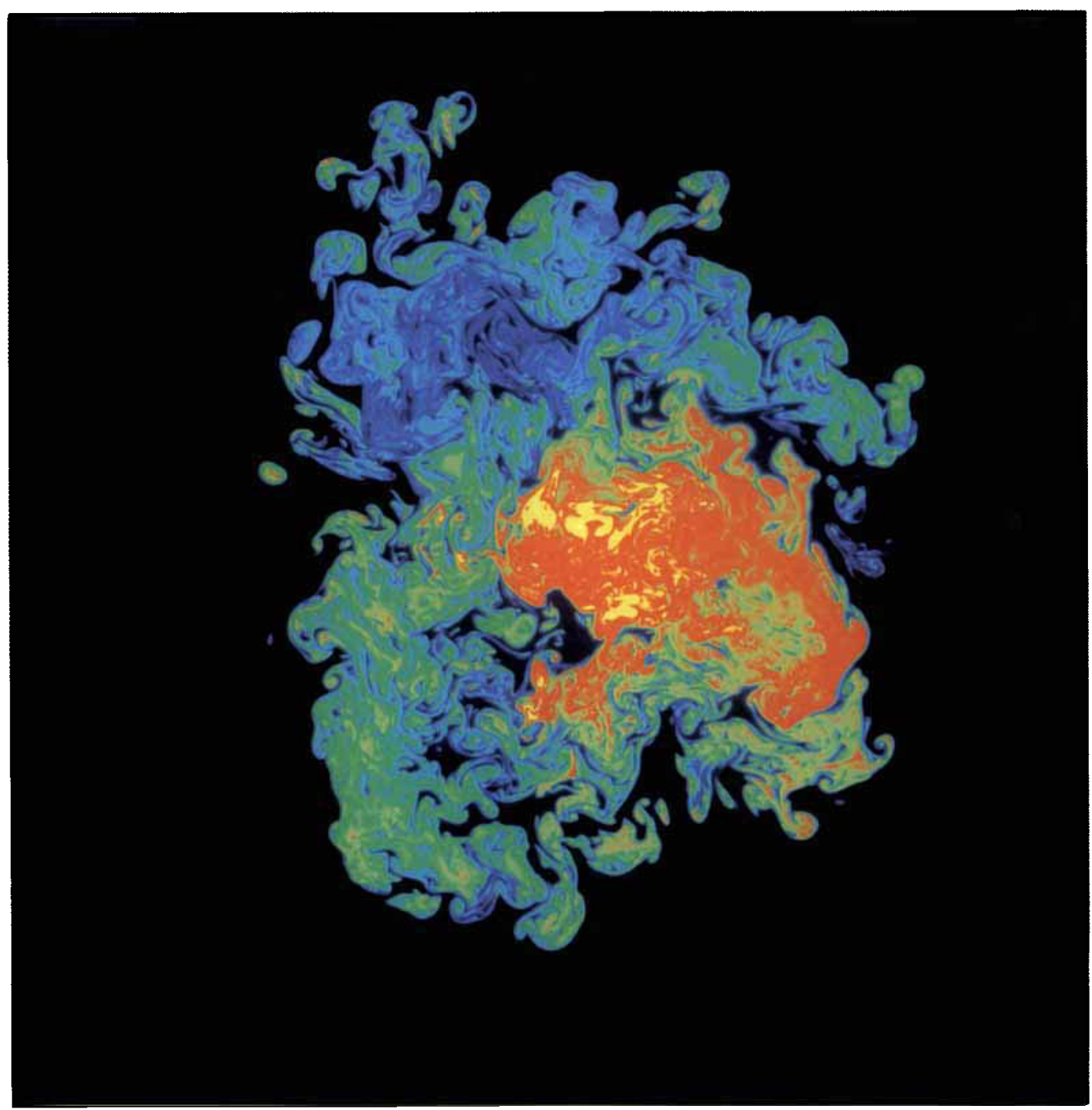

FIGURE 3. Jet-fluid concentration in the far field $\left(z / d_{0}=275\right)$ of a turbulent jet at $R e \simeq 4.5 \times 10^{3}$.

to the (pure) jet-plenum concentration, $c_{0}$, i.e.

$$
\frac{c(x, y)}{c_{0}}=\frac{c(x, y)}{c_{r e f}} \frac{c_{r e f}}{c_{0}}=\frac{c(x, y)}{c_{r e f}} \frac{V_{\text {plenum }}}{\left(V_{\text {tank }}+V_{\text {plenum }}\right)} \simeq \frac{c(x, y) / c_{r e f}}{2.2 \times 10^{2}},
$$

where $V_{\text {plenum }} \simeq 5.0 \times 10^{-3} \mathrm{~m}^{3}$ is the plenum volume and $V_{\text {tank }} \simeq 1.1 \mathrm{~m}^{3}$ is the tank volume.

The jet Reynolds number was varied in this investigation in the range $4.5 \times 10^{3} \leqslant$ $R e \leqslant 18 \times 10^{3}$. For round momentum-driven turbulent jets, at a downstream distance $z, \operatorname{Re} \equiv u(z) \delta(z) / v$, where $u(z) \propto 1 / z$ is the local jet centreline velocity, $\delta(z) \propto z$ is the local jet transverse extent (diameter), and $R e$ is independent of the downstream coordinate; $R e \simeq R e_{0}$, where $R e_{0} \equiv u_{0} d_{0} / v$ is the Reynolds number based on jetnozzle values. Examples of turbulent-jet scalar-image data at $R e \simeq 4.5 \times 10^{3}, 9.0 \times 10^{3}$, and $18 \times 10^{3}$, normalized and calibrated (cf. equation (2.1)), are depicted in figures 3 , 4 , and 5 , respectively. 


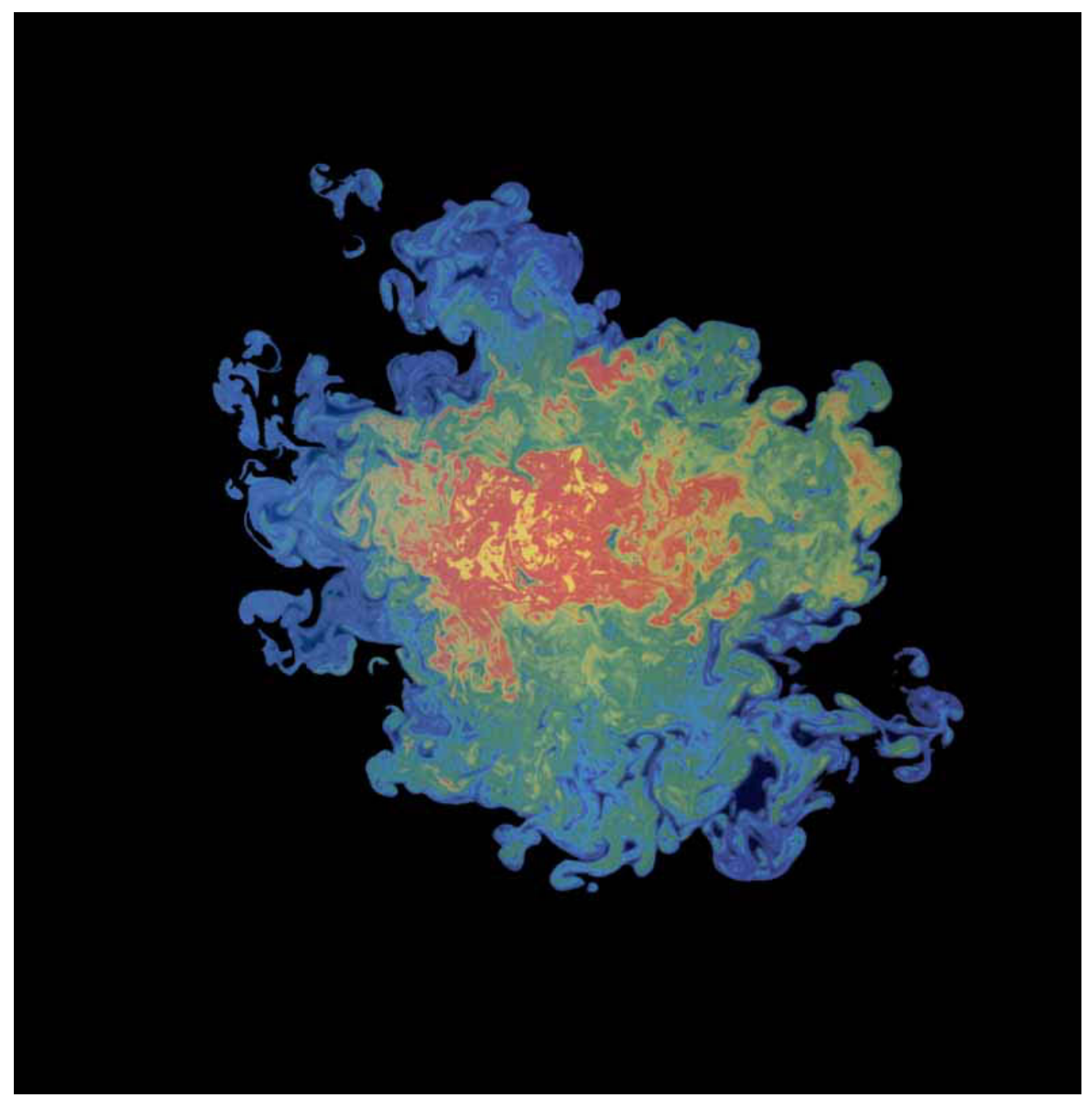

Figure 4. Jet-fluid concentration in the far field $\left(z / d_{0}=275\right)$ of a turbulent jet at $\operatorname{Re} \simeq 9.0 \times 10^{3}$.

Throughout the Reynolds number range investigated, the image field of view was the same. At a Reynolds number of $R e \simeq 9.0 \times 10^{3}$ (cf. figure 4), scalar diffusionlayer thickness scales (half-wavelength) on the jet centreline are estimated to be approximately half the pixel resolution, and much larger than the pixel resolution near the outer region of the jet. Also, at this Reynolds number, the time for the passage of these scales is estimated to be a factor of 30 times longer than the exposure time of an individual pixel, on the jet axis, and even longer near the boundary of the jet. The images were acquired maintaining a constant product of the beam-scanning time, which scaled the time exposure per pixel, and the local flow velocity, over the Reynolds numbers investigated. These choices provided temporally as well as spatially resolved measurements of the scalar field, throughout the Reynolds number range, certainly in the outer region of the jet where these investigations were primarily focused, with minor compromises in spatial resolution in the vicinity of the highest-velocity/-concentration (interior) regions of the jet. 


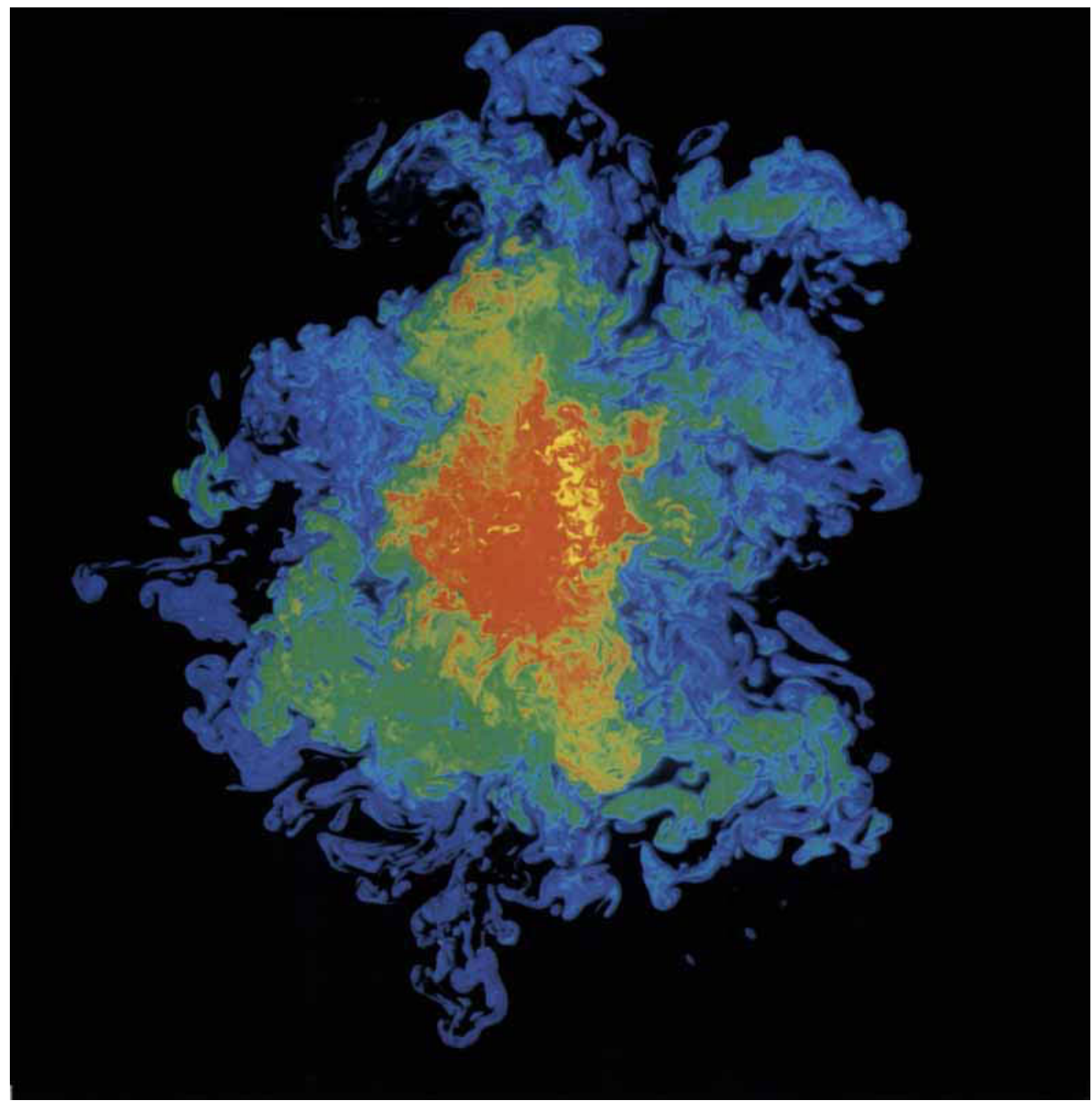

FIGURE 5. Jet-fluid concentration in the far field $\left(z / d_{0}=275\right)$ of a turbulent jet at $R e \simeq 18 \times 10^{3}$.

The two-dimensional scalar power spectrum for the image data of figure 4 is shown in figure 6. Contour values shown are in the range from -8.5 to -6.0 (outer to inner) in increments of 0.5 , in units of $\log _{10}\left(\ell_{0}^{2} c_{r e f}^{2}\right)$. The contours are seen to be approximately circular, consistent with a statistically axisymmetric scalar field. These spectra have been normalized such that the integral (sum) over the two-dimensional wavenumber space recovers the spatially averaged scalar variance, $\left\langle c^{2}\right\rangle$, which was estimated directly from the image data.

Radial scalar power spectra, $S_{c}\left(\kappa \ell_{0}\right)$, where $\kappa=\left(\kappa_{x}^{2}+\kappa_{y}^{2}\right)^{1 / 2}=k / 2 \pi$ is the radial wavenumber, for the three Reynolds numbers investigated (ensemble-averaged from six individual-image realizations at $R e \simeq 9.0 \times 10^{3}$, and three realizations at $R e \simeq$ $4.5 \times 10^{3}$ and $R e \simeq 18 \times 10^{3}$ ), are shown in figure 7 . These were estimated by an azimuthal constant- $\kappa$ (shell) integration of the corresponding two-dimensional scalar power spectra (cf. figure 6).

These spectra reflect the spatial confinement of the jet-fluid concentration field, 


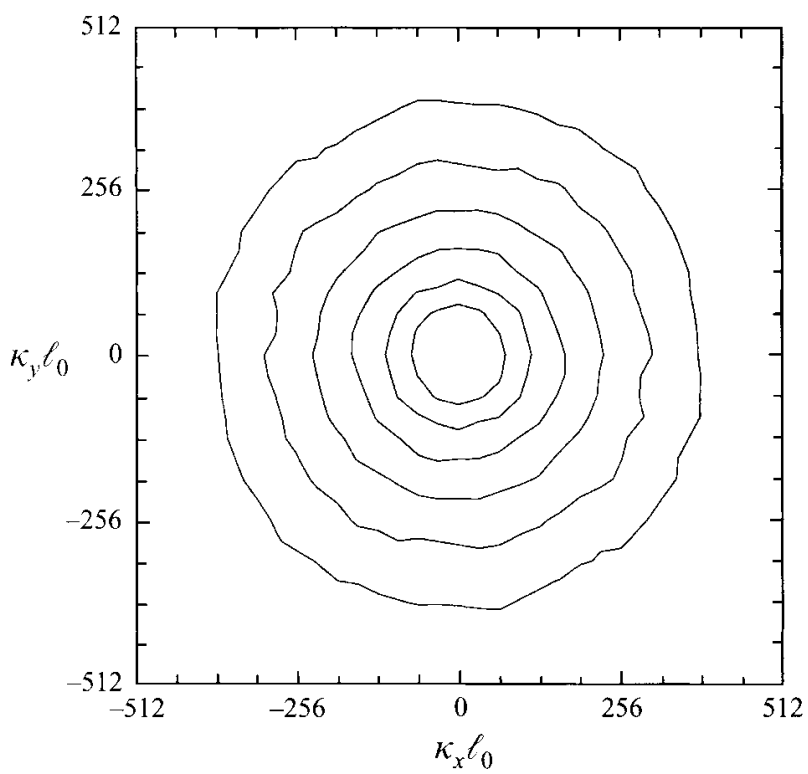

Figure 6. Contour plot of the (decimal) logarithm of the two-dimensional, spatial scalar power spectrum of the image data depicted in figure $4\left(\ell_{0}=42 \mathrm{~cm}\right.$ denotes the spatial extent of the field of view).

which is not a spatially (statistically) homogeneous field. Also noteworthy is the decrease, at high wavenumbers, with increasing Reynolds number, as will be discussed below. This is not a consequence of inadequate resolution; the spatial spectra diverge at rather low wavenumbers, with a separation that does not appreciably increase with wavenumber and, in particular, is not characteristic of fixed-pole low-pass filtering. The dynamic range of the scalar image data can be seen to be, approximately, $50 \mathrm{~dB}$, corresponding to a signal-to-noise (amplitude) ratio of 300:1.

\section{Classical scalar and isoscalar measures}

The jet-fluid-concentration probability-density function (p.d.f.), estimated as a histogram of the scalar values over the imaged field, is shown in figure 8 for the Reynolds numbers investigated. This was computed from ensemble-averaged histograms of six scalar images for $R e \simeq 9.0 \times 10^{3}$ and from three scalar images for $R e \simeq 4.5 \times 10^{3}$ and $R e \simeq 18 \times 10^{3}$ (cf. figures 3, 4, and 5). Lines of increasing solidity denote increasing Reynolds number. The scalar values are normalized by a constant reference concentration, $c_{r e f}$, where $c_{\text {ref }} / c_{0} \simeq 2.2 \times 10^{2}$ (cf. equation (2.2)). Low scalar values in figure 8 are generally encountered in the outer portion of the images (tank fluid), while high values are encountered in the interior of the images near the jet centreline, as indicated in figures 3,4 , and 5 .

Figure 8 shows that, in the Reynolds number range investigated, the shape of the scalar p.d.f. changes qualitatively with increasing Reynolds number. The peak of the mixed-fluid concentration p.d.f. shifts to lower values, with a relatively welldefined mixing transition occurring between $R e \simeq 9.0 \times 10^{3}$ and $R e \simeq 18 \times 10^{3}$. The p.d.f. values depicted in figure 8 are normalized over the field of view, which is the same for all images. Figure 8 shows that the (mixed-fluid) p.d.f. values increase with increasing Reynolds number. This implies that the (mixed) jet-fluid concentration 


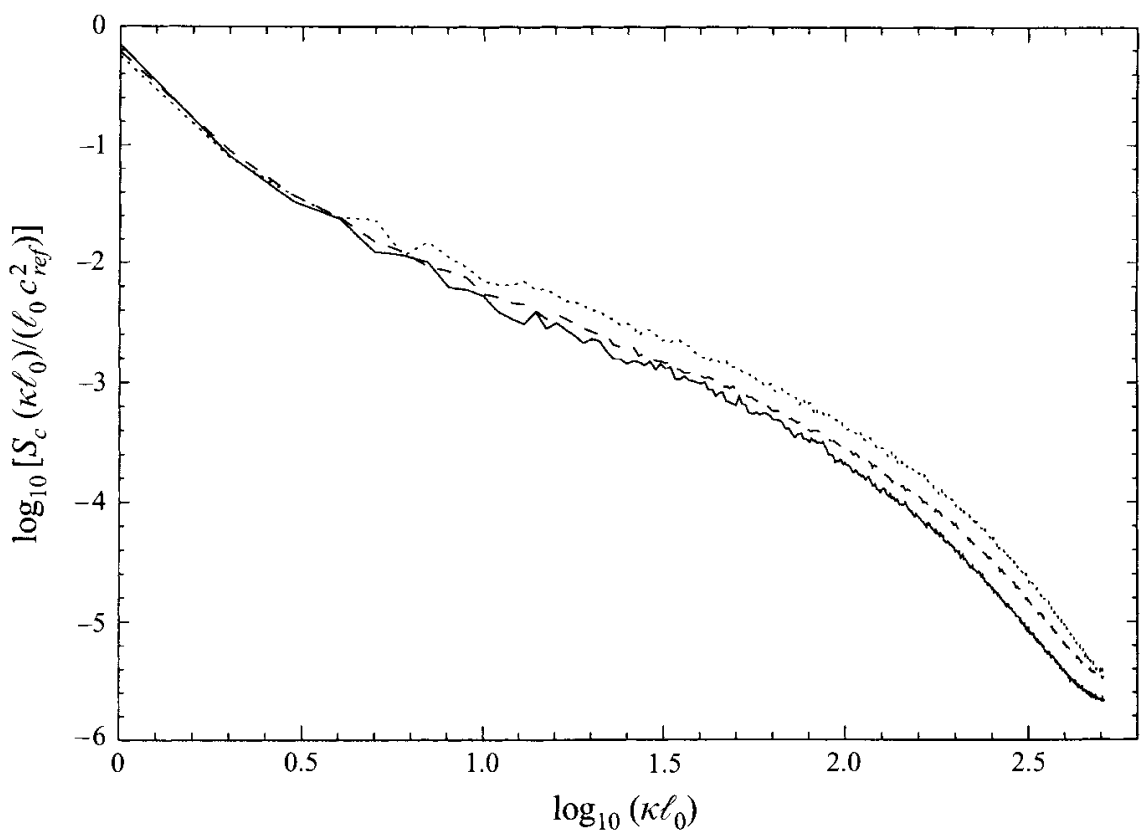

FIGURE 7. Ensemble-averaged radial scalar power spectra. $R e \simeq 4.5 \times 10^{3}:$ dotted line; $R e \simeq 9.0 \times 10^{3}:$ dashed line; $R e \simeq 18 \times 10^{3}:$ solid line.

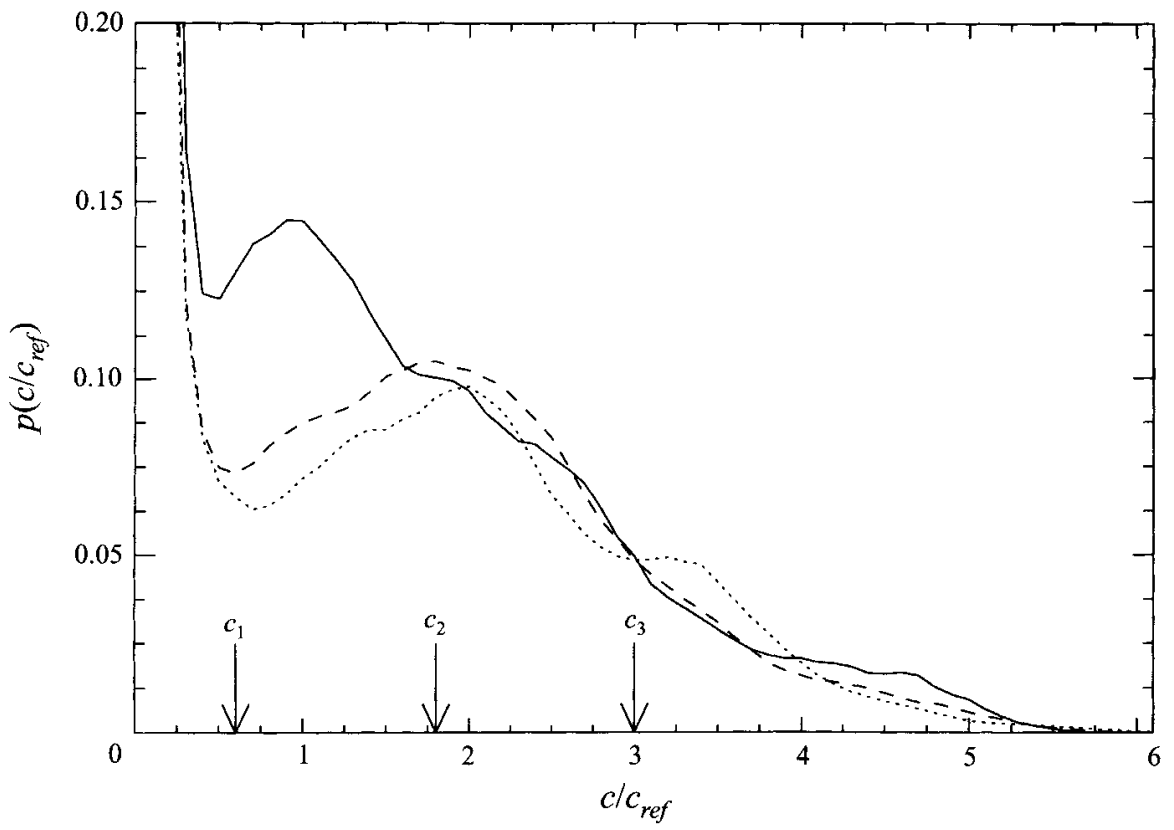

FIGURE 8. Jet-fluid concentration p.d.f. in the far field $\left(z / d_{0}=275\right)$ of a turbulent jet, as a function of Reynolds number. $R e \simeq 4.5 \times 10^{3}$ : dotted line; $R e \simeq 9.0 \times 10^{3}:$ dashed line; $R e \simeq 18 \times 10^{3}:$ solid line. Three scalar threshold values, $c_{1}, c_{2}$, and $c_{3}$, are also indicated. 

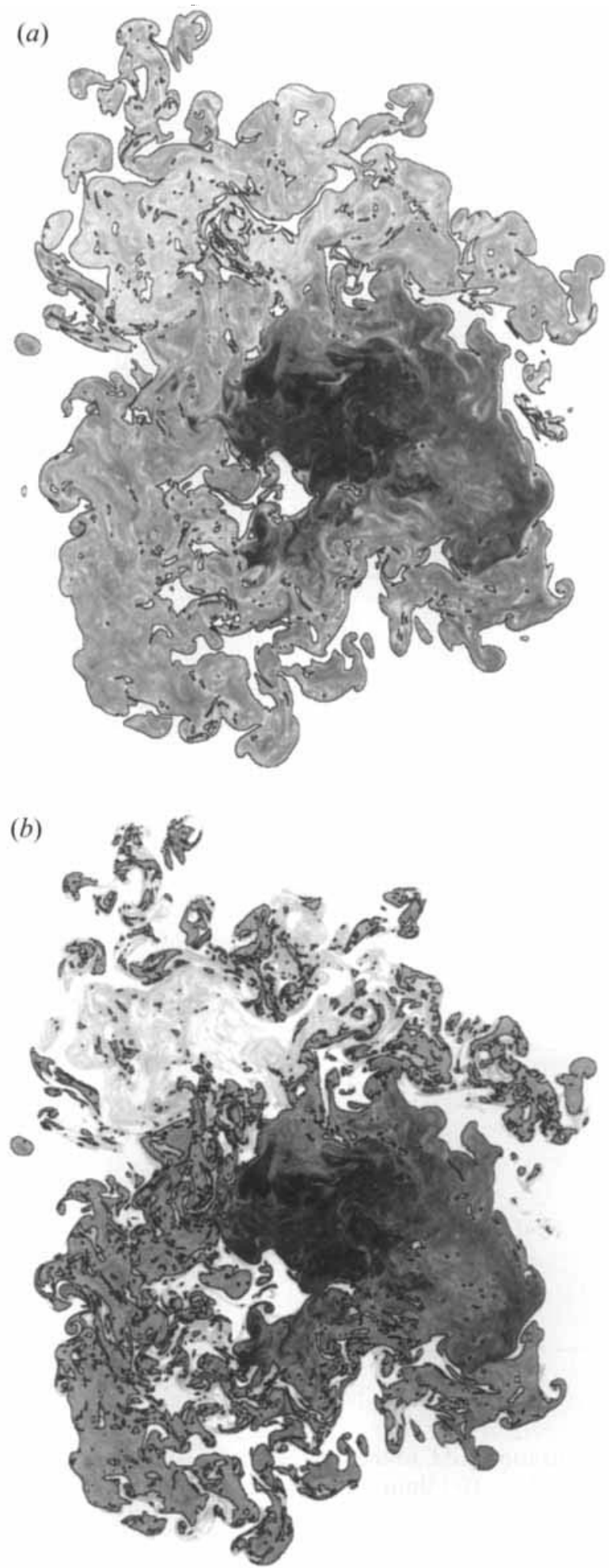

FIGURE $9(a, b)$. For caption see facing page. 


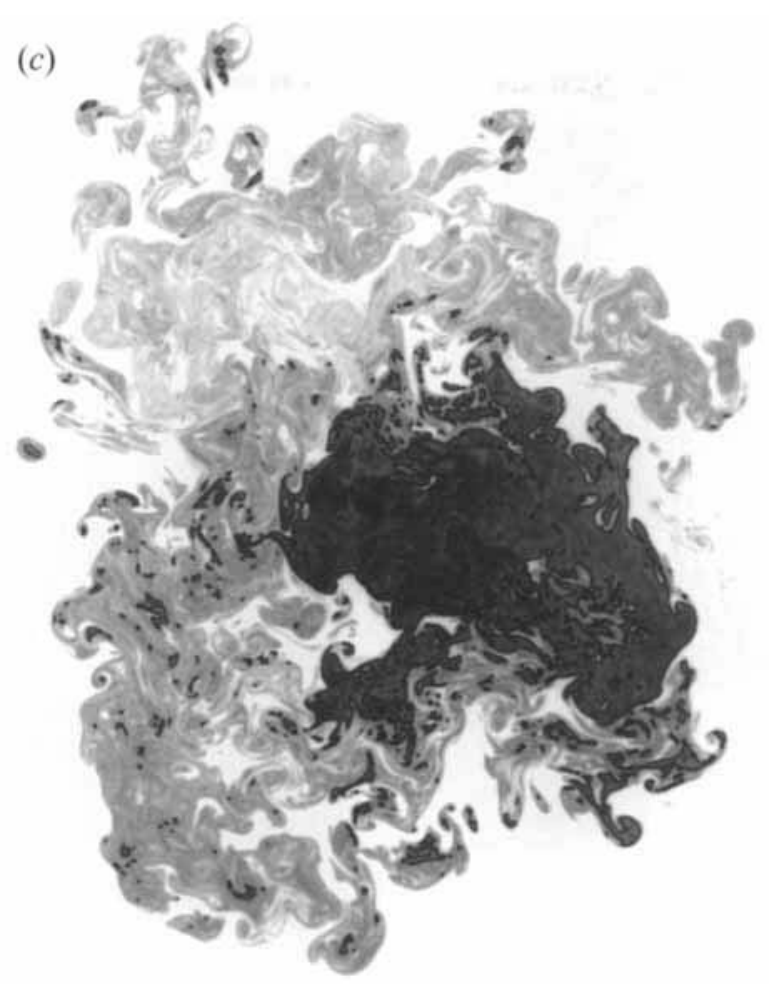

FiguRE 9. Isoscalar surface at $R e \simeq 4.5 \times 10^{3}$, superimposed on the image data of figure 3 .

(a) Threshold level, $c=c_{1},(b) c=c_{2},(c) c=c_{3}$.

scalar field covers a larger fraction of the imaged region with increasing Reynolds number. The change in the nature of the scalar p.d.f. with increasing Reynolds number, evident in figure 8 , occurs in the same range of Reynolds numbers which has been documented to produce a transition to fully developed turbulence in jets as well as other flows (cf. Dimotakis 1993).

The scalar-p.d.f. behaviour depicted in figure 8 indicates that isoscalar measures can be expected to be threshold as well as Reynolds-number dependent. In the subsequent analysis, three representative scalar thresholds have been chosen for the computation of scalar and isoscalar measures. These values are $c_{1} / c_{\text {ref }}=0.6, c_{2} / c_{\text {ref }}=1.8$, and $c_{3} / c_{\text {ref }}=3.0$, as indicated in figure 8 . The intermediate threshold, $c_{2}$, was chosen to correspond to the peak of the pre-transition p.d.f.'s, i.e. at $R e \simeq 9.0 \times 10^{3}$ and $R e \simeq 4.5 \times 10^{3}$. The lowest threshold, $c_{1}$, corresponds to the outer isosurfaces for all Reynolds numbers in the range investigated and to the vicinity of the local minimum of the p.d.f. for the lower Reynolds numbers, for which PLF behaviour has been reported (cf. Sreenivasan 1991). The high threshold, $c_{3}$, was chosen to investigate high-level behaviour, mindful of avoiding potential spatial resolution limitations that could be encountered at higher levels yet.

Examples of isoscalar surfaces (contours) at $R e \simeq 4.5 \times 10^{3}$, for the three scalar thresholds, $c_{1}, c_{2}$, and $c_{3}$, are shown in figures $9(a)-9(c)$, respectively. The isosurfaces are superimposed on the image data of figure 3, from which they are derived. Figure $10(a-c)$ and figure $11(a-c)$ show isoscalar surfaces at $R e \simeq 9.0 \times 10^{3}$ and $R e \simeq 18 \times 10^{3}$, respectively, for scalar thresholds $c_{1}, c_{2}$, and $c_{3}$. These isoscalar 


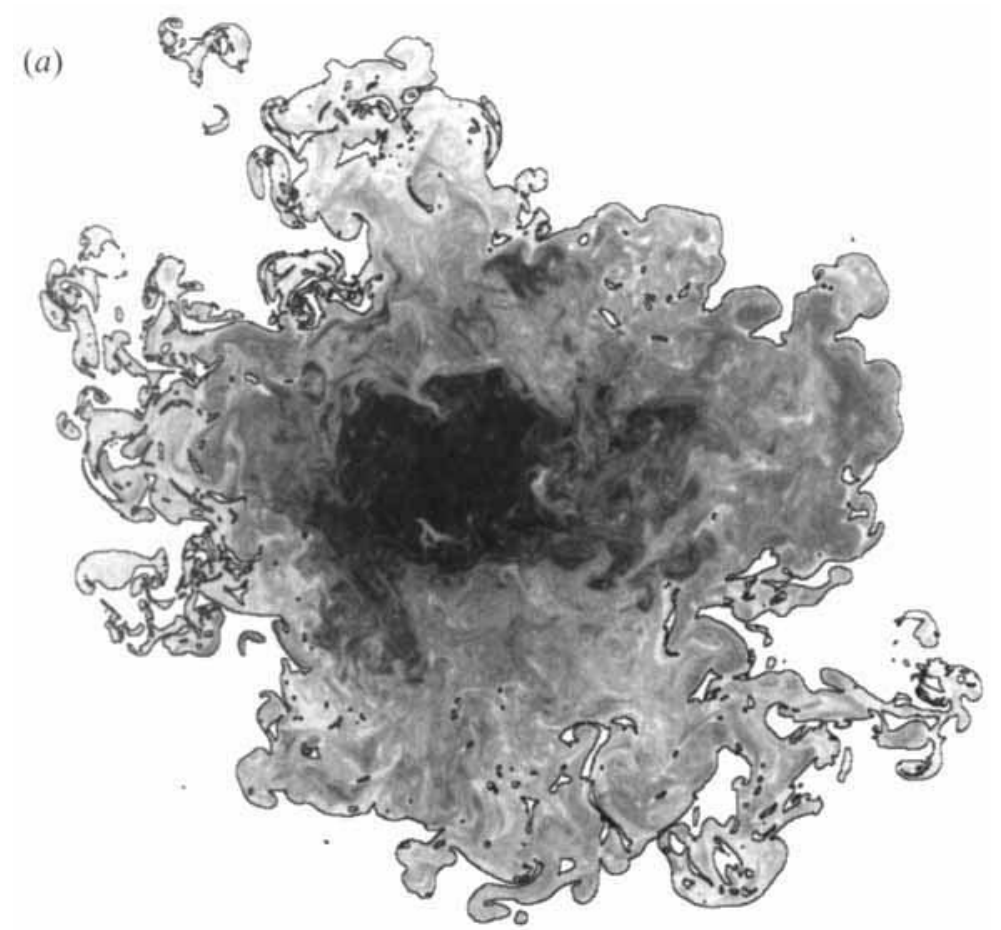

(b)

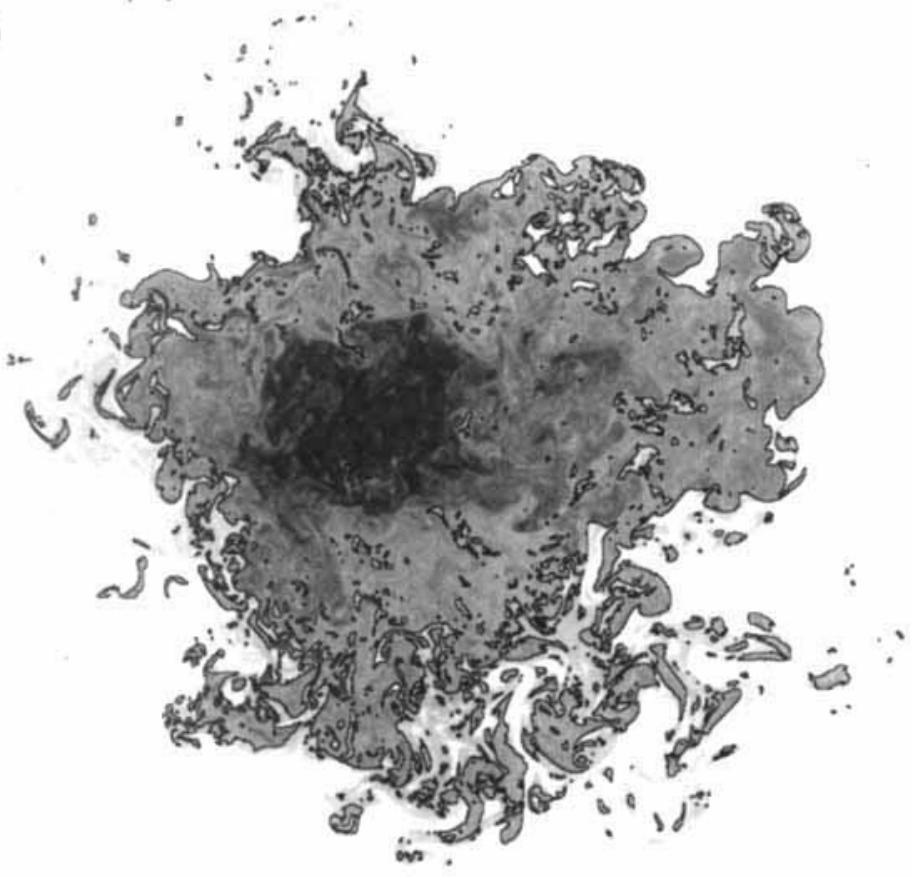

Figure $10(a, b)$. For caption see facing page. 


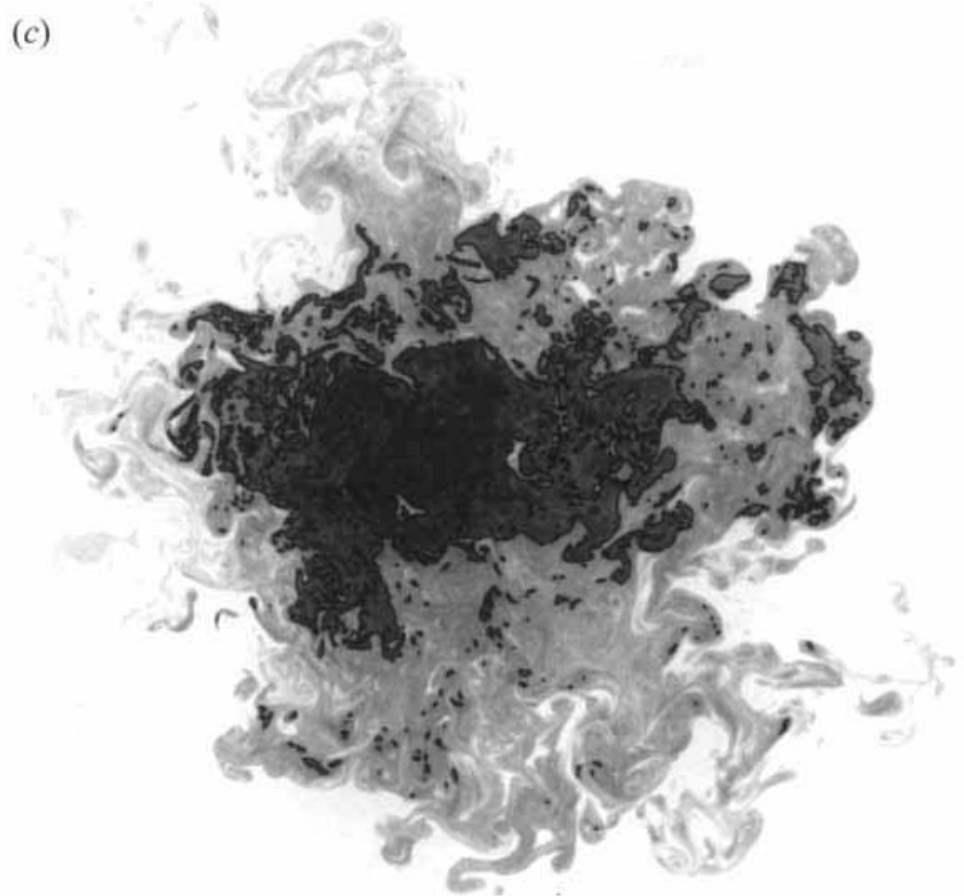

FIGURE 10. Isoscalar surface at $R e \simeq 9.0 \times 10^{3}$, superimposed on the image data of figure 4 . (a) Threshold level, $c=c_{1},(b) c=c_{2},(c) c=c_{3}$.

surfaces were constructed from the measured scalar-field data using bilinear B-splines and are depicted, in these figures, using boundary-outline pixels (cf. Appendix).

The area enclosed by the isoscalar surfaces, at a level $c$, scaled by the square of the distance downstream, i.e. $A(c) / z^{2}$, is shown in figure 12 , as a function of scalar threshold and Reynolds number. This quantity was computed as the summation of the area of all regions of the scalar field with values larger than or equal to the threshold, $c$. For the computation of this area, the isosurfaces were represented using bilinear B-splines (cf. Appendix). In the case of non-premixed hydrocarbon jet flames, for example, this area measure would represent the fraction of the cross-section occupied by unburnt jet fluid, e.g. fuel, for a scalar level chosen to coincide with the stoichiometric fuel-to-air mixture fraction.

The area measure, $A(c)$, is an integral measure of the scalar field, representing the imaged cross-section where jet fluid has not yet mixed down to the level, $c$. It can also be expressed as an integral of the spatially normalized p.d.f. of scalar values, i.e.

$$
A(c) / A_{0}=\int_{c}^{\infty} p\left(c^{\prime}\right) \mathrm{d} c^{\prime}
$$

where $A_{0}=\ell_{0}^{2}$ is the total area of the image field (this consistency requirement was confirmed with the two expressions independently estimated from the image data, as described above). Equation (3.1) is equivalent to the geometric interpretation of the scalar p.d.f., in terms of the differential area (volume) between isosurfaces separated by a differential scalar interval, i.e. for these two-dimensional data, $p(c) \mathrm{d} c=|\mathrm{d} A(c)| / A_{0}$ (e.g. Kuznetsov \& Sabel'nikov 1990, p. 27).

The area enclosed by the outer isosurfaces, at a given threshold, is seen to increase 


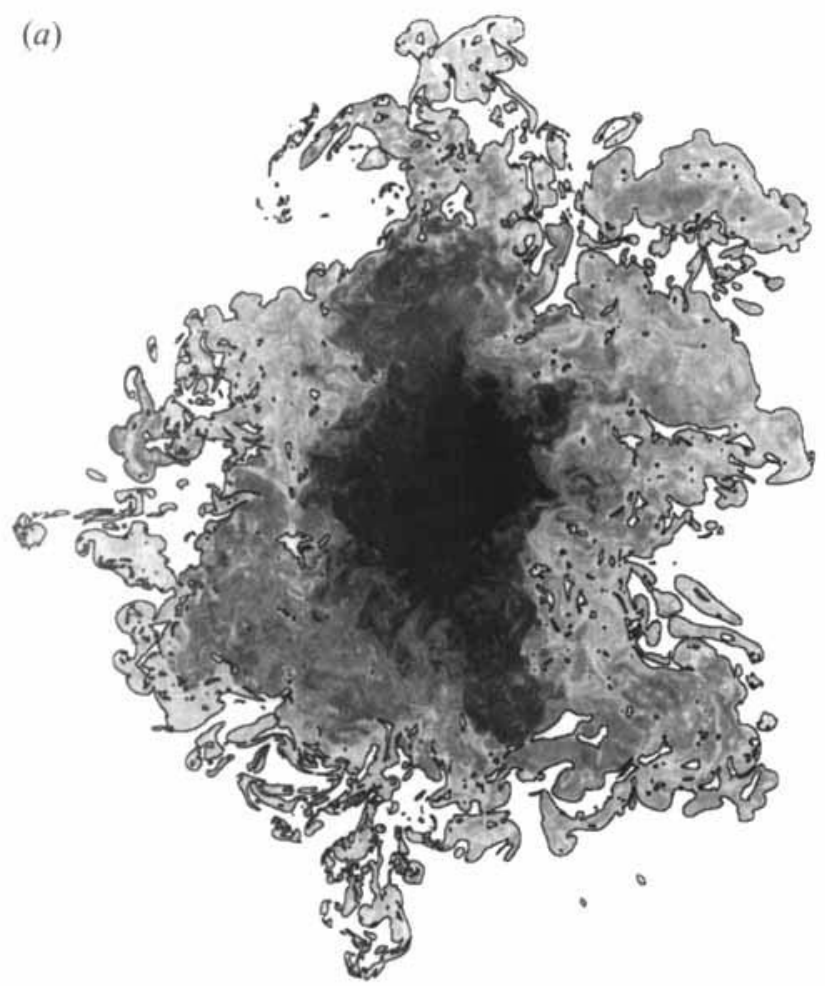

(b)

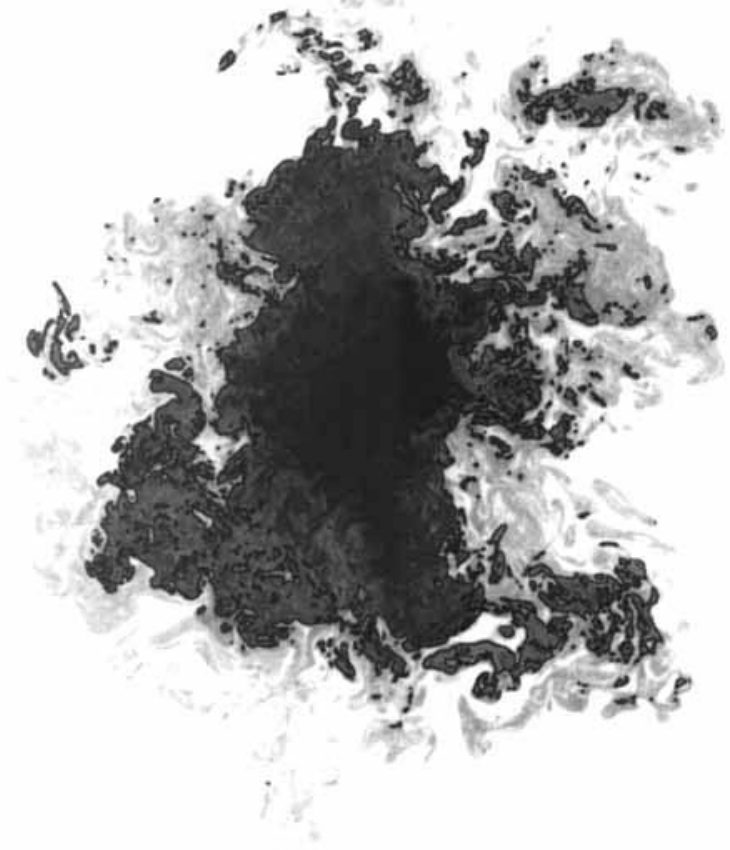

Figure 11 $(a, b)$. For caption see facing page. 


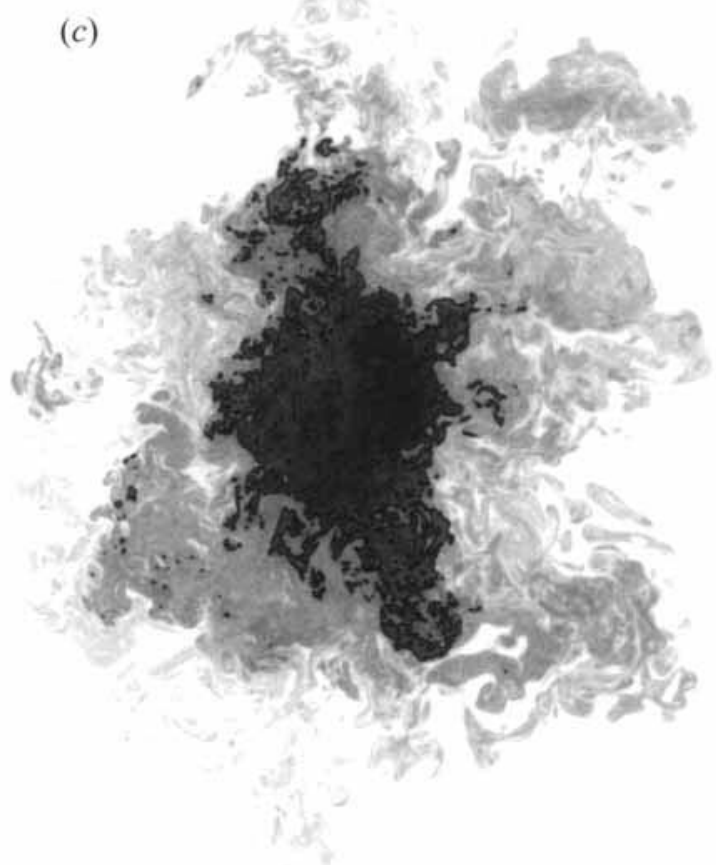

FIGURE 11. Isoscalar surface at $R e \simeq 18 \times 10^{3}$, superimposed on the image data of figure 5 . (a) Threshold level, $c=c_{1},(b) c=c_{2},(c) c=c_{3}$.

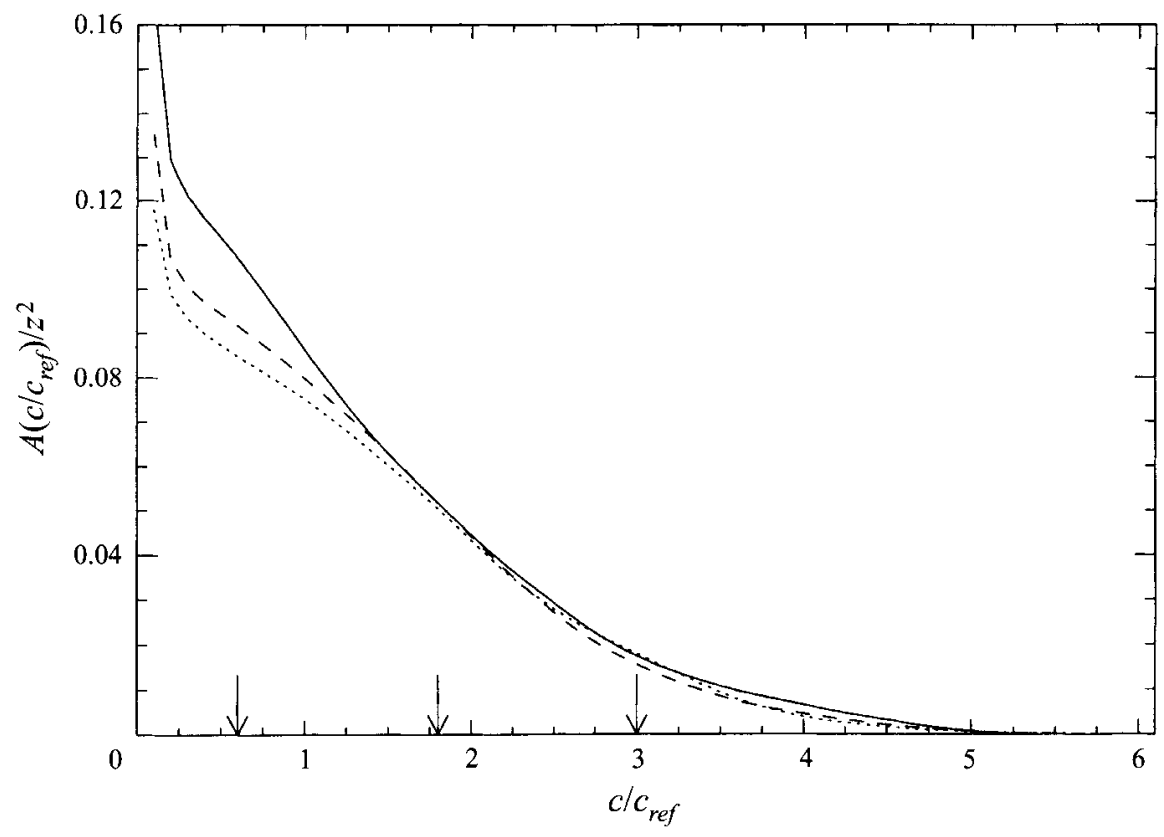

FIGURE 12. Area, $A$, enclosed by isoscalar surfaces as a function of scalar threshold and Reynolds number. $R e \simeq 4.5 \times 10^{3}:$ dotted line; $R e \simeq 9.0 \times 10^{3}:$ dashed line; $R e \simeq 18 \times 10^{3}:$ solid line. Arrows label $c_{1}, c_{2}$, and $c_{3}$ scalar threshold values (cf. figure 8). 


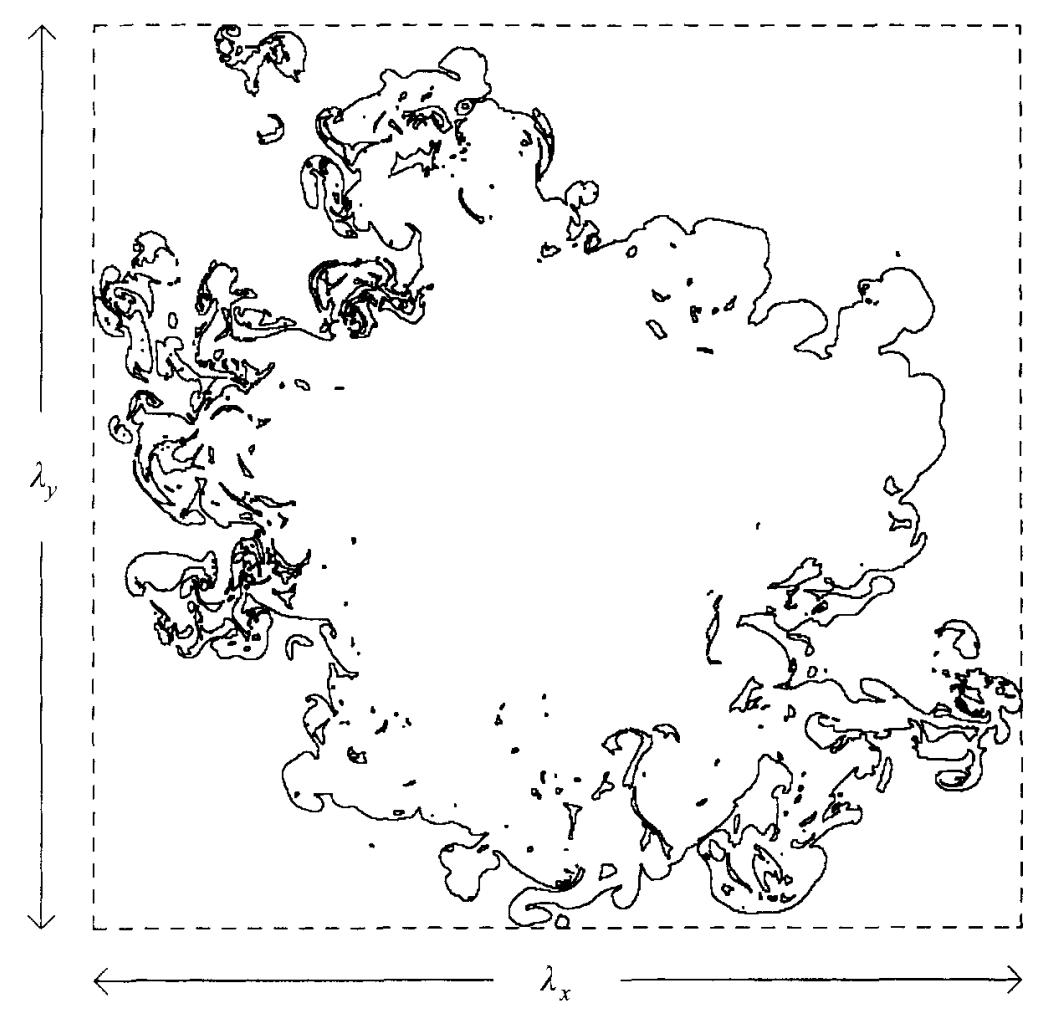

FIGURE 13. Bounding box (dashed line) for the level set of figure 10(a).

Dotted line: field-of-view boundaries.

with increasing Reynolds number. Additionally, this behaviour is seen to occur in a range of lower thresholds that diminishes with increasing Reynolds number. Conversely, higher threshold regions do not exhibit conspicuous Reynolds number effects, with variations well within our statistical confidence for this measure. These observations may be explained by appreciating that lower-scalar-level isosurfaces are generally encountered in the outer regions of the jet (cf. figures 9,10 , and 11), where lower velocities, increased viscous effects, and higher sensitivity to the flow Reynolds number can be expected.

The extent of the scalar isosurfaces (cf. figures 9, 10 and 11) allows the local mixed-fluid extent to be estimated. This can be computed by identifying the bounding box that encloses each isosurface, in general, or the smallest circumscribing rectangle (cf. Tricot 1995), in two dimensions. Figure 13 depicts such a bounding box, identified for the isosurface in figure $10(a)$, as well as the extent of the field of view. Note that, while this rectangle is oriented along the field-of-view axes, the near-circular 


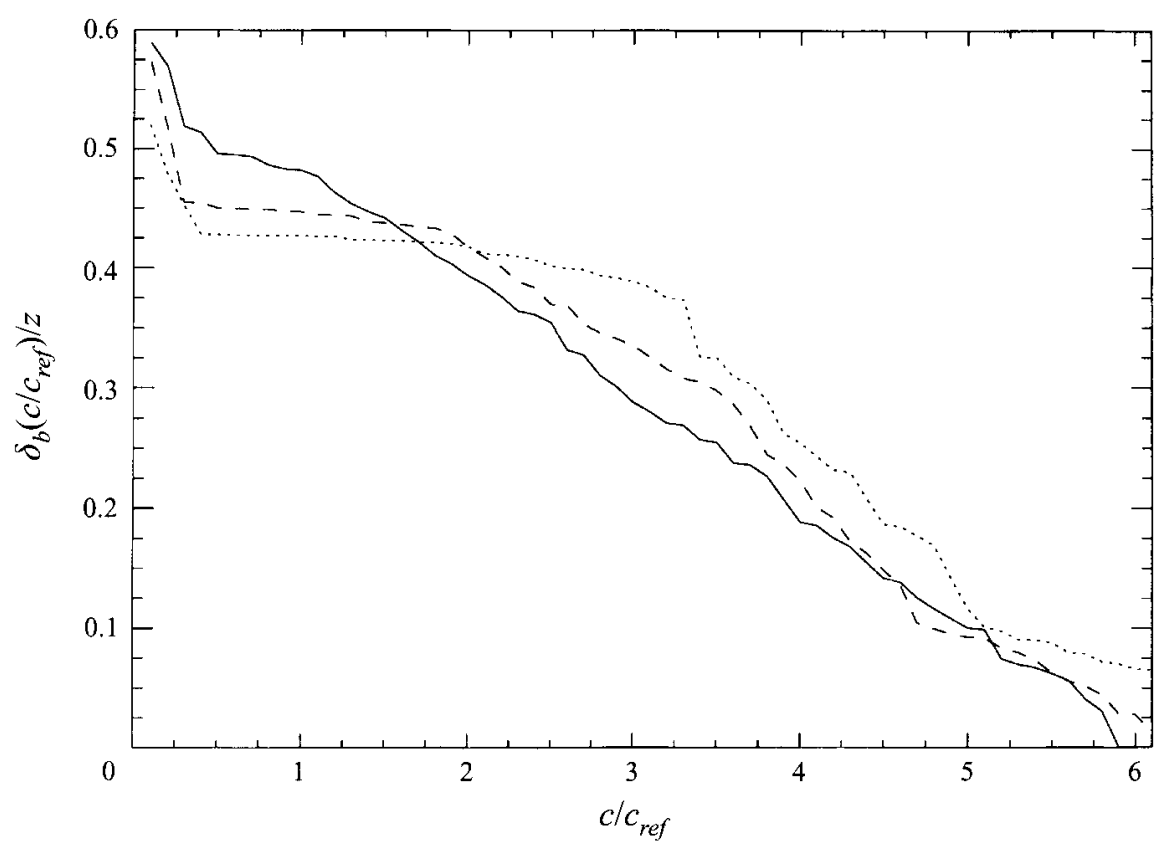

FIGURE 14. Isoscalar spatial extent, $\delta_{b}$, based on bounding box, as a function of scalar threshold and Reynolds number. $R e \simeq 4.5 \times 10^{3}$ : dotted line; $R e \simeq 9.0 \times 10^{3}$ : dashed line; $R e \simeq 18 \times 10^{3}$ : solid line.

symmetry of the individual two-dimensional scalar power spectra (cf. figure 6), as well as the ensemble-averaging over separate realizations, renders it a useful measure. The isoscalar spatial extent, $\delta_{b}$, was computed as the square root of the area of the rectangle (bounding box), i.e.

$$
\delta_{b} \equiv\left(\lambda_{x} \lambda_{y}\right)^{1 / 2},
$$

where $\lambda_{x}$ and $\lambda_{y}$ are the lengths of the two sides of the circumscribing rectangle; cf. equation (A 1) and related discussion.

Figure 14 shows the ensemble-averaged spatial extent scaled by the distance downstream, $\delta_{b} / z$, as a function of scalar threshold and Reynolds number. Alternatively, $\delta_{b} / 2$ is a measure of the maximum distance from the jet axis where a particular scalar value will be encountered. As expected, the isoscalar spatial extent, $\delta_{b}$, decreases monotonically with increasing scalar threshold, at a given Reynolds number. As with the scalar p.d.f.'s and the area measure, a qualitative change in the dependence of $\delta_{b}$ on the scalar value, $c / c_{r e f}$, can be seen as the Reynolds number is increased from $4.5 \times 10^{3}$ to $18 \times 10^{3}$. The spatial extent of the outer isosurfaces increases with increasing Reynolds number, while the extent of the inner isosurfaces decreases with increasing Reynolds number. In addition, the variation of $\delta_{b}$ with $c / c_{r e f}$ appears to approach a straight line as the Reynolds number is increased (cf. figure 14), i.e.

$$
\frac{\delta_{b}}{z} \longrightarrow \alpha-\beta \frac{c}{c_{0}},
$$

with $\alpha \simeq 0.6$ and $\beta \simeq 0.1 c_{0} / c_{r e f} \simeq 22$; cf. equation (2.2). This behaviour, in addition to the changes in the nature of the scalar p.d.f.'s and area enclosed by the level sets noted above, provides further evidence of a mixing transition in this range of Reynolds numbers. 
At the intermediate scalar threshold, $c_{2}$, corresponding to the peak of the pretransition p.d.f.'s, the isoscalar spatial extent, $\delta_{b}$, is seen to be (approximately) Reynolds-number independent (figure 14), at least for the Reynolds numbers investigated. This feature will be exploited in the analysis of Reynolds-number effects on the geometry of the isoscalar surfaces, i.e. comparisons of geometric measures, at different Reynolds numbers, will be made at this intermediate threshold.

\section{Power-law-fractal (PLF) geometry}

Ever since Richardson's (1961) analysis of data on the length of coastlines and borders between countries and Mandelbrot's (1967) subsequent interpretation and proposals, it has become appreciated that convoluted curves and surfaces can be regarded as possessing scale-dependent length and area, respectively, that increase as the measurement resolution is extended to smaller and smaller scales. This is a consequence of the presence of ever-finer features that are encountered as the scale of measurement is reduced, in many phenomena. One particular form of this scale dependence is a power-law function. The resulting fractal, or power-law-fractal (PLF), geometry has been proposed as an appropriate framework for the characterization of various complex structures in nature (e.g. Mandelbrot 1967, 1975b, 1977, 1982).

PLF curves in a plane, for example, have a coverage length, $L_{2}(\lambda)$, that increases in a power-law manner with decreasing scale, $\lambda$, i.e. (cf. Richardson 1961)

$$
L_{2}(\lambda) \propto \lambda^{1-D_{2}}
$$

(with a dimensional prefactor), where $1<D_{2} \leqslant 2$ is the PLF dimension. The subscript 2, here, denotes the two-dimensional embedding (Euclidean) space of these curves. The coverage length, $L_{2}(\lambda)$, is defined in terms of the (box) coverage, $N_{2}(\lambda)$, which is the number of (non-overlapping Euclidean) tiles (2-D boxes) of size $\lambda$ needed to cover the curve, i.e.

$$
L_{2}(\lambda) \equiv \lambda N_{2}(\lambda)
$$

where, for PLF curves, the coverage follows a power-law,

$$
N_{2}(\lambda) \propto \lambda^{-D_{2}},
$$

also, presumably, with a dimensional prefactor and a scaling exponent given by the (negative of the) constant PLF dimension, $D_{2}$.

In a $d$-dimensional embedding space, PLF sets are characterized by a geometric coverage, $N_{d}(\lambda)$, given by the number of (non-overlapping Euclidean) boxes (tiles, in 2-D), of size $\lambda$, needed to cover the set, that follows a power law, i.e.

$$
N_{d}(\lambda) \propto \lambda^{-D_{d}},
$$

also with a dimensional prefactor, where $d_{t}<D_{d} \leqslant d$ is the PLF dimension, and $d_{t}$ is the (integer-valued) topological dimension. PLF relations, such as equations (4.1), (4.3) and (4.4), describe scale-independent geometric-scaling laws. From such power-law relations, the PLF dimension is identified as the scaling exponent of the coverage, i.e.

$$
D_{d}=-\frac{\mathrm{d} \log N_{d}(\lambda)}{\mathrm{d} \log \lambda}
$$

in a range of scales. This PLF dimension can be expected to be constant if, and only if, the coverage, $N_{d}(\lambda)$, follows an exact power law, i.e. only for geometric structures possessing scale self-similarity. 
Mandelbrot $(1975 a, b)$ proposed that PLF geometry may be applicable to turbulence, suggesting a PLF dimension of $D_{3}=8 / 3$ for isoscalar surfaces in homogeneous turbulence with Kolmogorov-Gauss scaling. In the last ten years, or so, the geometry of isosurfaces in turbulence has been the object of various experimental, numerical, as well as theoretical studies. Several investigators have reported PLF behaviour in the geometry of turbulence. Sreenivasan \& Meneveau (1986) reported experimental findings of PLF dimensions, $D_{1}=0.32$ and $D_{2}=1.33$, for isoscalar measurements in a turbulent jet, from which they argued that $D_{3}=2.33$ for the (three-dimensional) isoscalar surfaces. Sreenivasan et al. (1989) found a PLF dimension of $D_{2}=1.36$ for isoscalar jet data, with $D_{2}=1.35 \pm 0.05$ as a mean value for various turbulent shear flows, and offered arguments for a value of $D_{3}=7 / 3$. Prasad \& Sreenivasan (1990) analysed three-dimensional data of the isoscalar surfaces in turbulent jets and reported a PLF dimension of $D_{3}=2.35 \pm 0.04$. A theoretical estimate for a PLF dimension of $D_{3}=2.5$ was obtained by Constantin $(1990,1991)$, later refined to $D_{3}=8 / 3$ (Constantin, Procaccia \& Sreenivasan 1991; Constantin 1994a,b; and Constantin \& Procaccia 1994). A lack of PLF scaling was noted by Sreenivasan (1991, p. 553), for isoscalar surfaces in the interior of the jet. Constantin et al. (1991), however, reported PLF scaling in the jet interior, with $D_{3}=2.67$, citing the same isosurface for which Sreenivasan (1991, p. 553) had originally concluded that there was no PLF scaling. In particular, Constantin et al. (1991) suggested PLF dimensions of $D_{3}=8 / 3$, for isoscalar surfaces in the jet interior, and $D_{3}=7 / 3$, for isoscalar surfaces near the jet boundary. Procaccia et al. (1992) pointed out, however, that "the theory [of Constantin et al. (1991)] cannot exclude the possibility that the scaling exponent $D$ depends on [the scale] $r$ " (inserts in square brackets ours). Procaccia et al. (1992) analysed isosurfaces of vorticity in three-dimensional homogeneous turbulence, using the direct-numerical-simulation data of Vincent \& Meneguzzi (1991), and concluded that, "... it is impossible to state with confidence that the [PLF] behaviour [of the vorticity isosurfaces] is clearcut." Lane-Serff (1993) reported a threshold-dependent PLF dimension of isoscalar surfaces in liquid-phase jet and plume flows, with a minimum value of $D_{2}=1.23$, computed by fitting a straight line using a least-squares fit. He noted, however, that "there is a distinct curve [i.e. curvature, in the coverage plots]" but attributed this to "the small range between integral and Kolmogorov scales at the Reynolds numbers of [the] experiments". Flohr \& Olivari (1994) analysed isoscalar surfaces in gas-phase turbulent jets and reported "constant [PLF] scaling behaviour over a wide range [of scales]" with a threshold-dependent PLF dimension exhibiting a maximum value. For the outer isoscalar surfaces, they suggested a PLF dimension of $D_{2}=1.30 \pm 0.05$. Sreenivasan (1994) suggested a PLF dimension of $D_{3}=2.35 \pm 0.05$ for outer isoscalar surfaces in turbulent jets, with PLF scaling "over much of the interval between the integral scale and the Kolmogorov scale," and a PLF dimension of $D_{3}=2.67 \pm 0.05$ for inner isoscalar surfaces in turbulent jets, in a scaling range "smaller" than that for the outer isosurfaces, indicating the degree of confidence of his results as "fairly certain".

Takayasu (1982) had argued, however, that the dynamics of turbulent flow varies with scale, and had suggested that, as a consequence, descriptions of the geometry of turbulence may be expected to require fractal dimensions that are functions of scale and not constant. Takayasu characterized the geometry of the path of a onedimensional random-walk particle with finite mean free path, using a scale-dependent fractal (SDF) dimension, and conjectured the applicability of SDF dimensions to turbulent diffusion. In random walks with finite mean free path, particles perform correlated, or inertial, random walks when observed at scales larger or smaller than the mean-free-path scale (Takayasu 1982). As a model of turbulent diffusion, Taylor 
(1921) had considered a modified random walk in which he allowed for particles with inertia, i.e. a random walk with correlated steps. Taylor's modification of the random walk illustrated the nature of turbulent diffusion as a correlated random walk (cf. McComb 1991). Borgas (1993) has offered Lagrangian-statistic arguments for a SDF dimension of particle trajectories in turbulent flow.

Miller \& Dimotakis (1991a) reported on experiments in the far field of liquid-phase turbulent jets, in which no PLF behaviour was found for scalar level sets derived from either point, line, or space-time (streak-image) measurements of the jet-fluidconcentration field, at least for thresholds in the vicinity of the mean of the scalar p.d.f. In particular, they found a SDF dimension, $D_{1}(\lambda)$, for scalar level sets derived from one-dimensional temporal and spatial data, with a smooth variation from 0 , at the smallest scales, to 1 , at the largest scales. They also reported values of $D_{1+1}(\lambda)$, increasing continuously with scale, from near 1 to almost 2 , for isoscalar contours derived from space-time data (one space dimension plus time), in the neighbourhood of the axis of turbulent jets. Sreenivasan (1991) commented on the Miller \& Dimotakis (1991a) findings, suggesting they could be attributable to differences between temporal and spatial data. See also Kerstein (1991) for an alternative discussion. Dimotakis (1991) argued, generally, that for scales $\lambda$ that are dimensional, as is the case here, there are dimensional and similarity issues that arise with expressions like equation (4.4) and that characteristic scales are necessary for PLF scaling, in contrast with previous proposals. Gluckman, Willaime \& Gollub (1993) conducted experiments in thermal turbulence and found that thermal isosurfaces do not display PLF scaling, while scalar isosurfaces show a limited range of "approximately-[power-law-]fractal" scaling.

\section{Scale-dependent-fractal (SDF) geometry}

A scale-dependent fractal (SDF) may be regarded as a geometric object characterized by a fractal dimension that is a function of the scale $\lambda$, i.e. $D_{d}(\lambda)$, in a range of scales. The subscript $d$ denotes the embedding (Euclidean) dimension for the object. SDF sets obey general geometric-scaling laws and allow for more complex geometric structures than PLF sets, which are, by definition, restricted to scale-invariant complexity. The difference between a PLF dimension, $D_{d}$, and a SDF dimension, $D_{d}(\lambda)$, can be illuminated by considering a hierarchy of complex patterns (Mikhailov \& Loskutov 1991). In particular, simple Euclidean objects, such as circles, spheres, have structure only at a certain (large) scale and are associated with the integer-valued embedding dimension, $d$. This level of complexity can be dubbed Level 1. PLF objects have structure that persists at all scales and, hence, are more complex than Level 1 objects; they can be assigned Level 2 in complexity. Level 2 objects are associated with a PLF dimension, $D_{d}$. For the PLF objects, it is the same structure that persists at various scales. A higher level of complexity, Level 3, can occur when the object has structure of variable complexity at different scales; this is the level of SDFs. Level 3 objects are characterized by a SDF dimension, $D_{d}(\lambda)$. SDFs can be seen, therefore, as scale-dependent generalizations of PLFs.

Several investigators have considered the notion of a SDF dimension, employing different terminologies to denote $D_{d}(\lambda)$, in various contexts. Some have also suggested models for SDF behaviour observed in a variety of natural phenomena. As noted above, Takayasu (1982) found SDF behaviour in characterizing the geometry of the path of random-walk particles. Using a real-space renormalization argument, he derived an expression for the successive coverage of a one-dimensional random walk with finite mean-free path. Takayasu initially used the term "differential fractal 
dimension," later employing the term "scale-dependent fractal dimension" (Takayasu 1992). Suzuki (1984) reported SDF behaviour for Japanese coastlines and suggested a SDF Koch curve model. Suzuki used the terms "transient fractal dimension" and "transient fractals" to describe SDF properties. Mark \& Aronson (1984) reported SDF behaviour in the analysis of topographic surfaces and used the term "scaledependent fractal dimension." Chilés (1988) studied fractured rocks and reported a continuously varying "local similarity dimension" as a function of scale. Chilés suggested several models for SDF behaviour, including SDF Cantor dust. A smooth variation of the coverage dimension with scale was found by Miller \& Dimotakis $(1991 a)$ in their analysis of turbulent-jet scalar data, as noted above. In characterizing the distribution of galaxies in the universe, Castagnoli \& Provenzale (1991) suggested that, "... it is probably necessary to consider models whose scaling and fractal properties vary with the spatial scale." In addition, Brandt et al. (1991), in their analysis of solar granulation data, found, "... a smooth transition of the fractal dimension from small to large granules." Rigaut (1991) studied surfaces of biological tissues, using microscopic biometry, and also reported SDF behaviour. In an analysis of the alveolar geometry of lungs of prematurely born rabbits, he reported a "drifting fractal dimension" with scale and used the term "semi-fractals" for SDFs. The term "scale-dependent fractal dimension", employed by Mark \& Aronson (1984) and Takayasu (1992), has been adopted here. It is appropriate, in our opinion, to call the resulting objects "scale-dependent fractals" (SDFs), since the word "fractal", coined by Mandelbrot $(1975 a, b)$ from the Latin fractus, means "fragmented", as he notes (Mandelbrot 1982, p. 4), and need not be excluded from referring to scale-dependent (fragmented) behaviour.

Consider a set, $\mathscr{S}$, embedded in a $d$-dimensional space, $E_{d}$, and contained in a $d$-dimensional bounding box of size $\delta_{b}$. Consider, also, successive partitions of the $\delta_{b}$-box into non-overlapping $\lambda$-size interior boxes that fill the $\delta_{b}$-box volume (cf. the Appendix for the two-dimensional implementation adopted here). The geometric (box) coverage, $N_{d}(\lambda)$, of the set, $\mathscr{S}$, is defined as the minimum number of non-overlapping partition $\lambda$-boxes needed to cover the set. We will then have

$$
\mathscr{S} \subset \bigcup_{i=1}^{N_{d}(\lambda)} B_{d}^{(i)}(\lambda),
$$

where $B_{d}(\lambda)$ denotes a $d$-dimensional box, interior to the bounding box, of size $\lambda$, i.e. of volume $\lambda^{d}$, with $B_{d}^{(i)}(\lambda) \cap B_{d}^{(j)}(\lambda)=\emptyset$, for $i \neq j$. The $d$-dimensional volume (area in two dimensions) of the portion of space visited by the set, at the coverage scale $\lambda$, is therefore $\lambda^{d} N_{d}(\lambda)$.

The notions of coverage measure, and capacity dimension, introduced by Hausdorff (1919) and Kolmogorov \& Tihomirov (1959), were defined in the limit of $\lambda \rightarrow 0$. Equation (5.1), however, allows the study of coverage statistics at any (finite) coverage scale, $\lambda$. In particular, the specification of non-overlapping partition boxes, in equation (5.1), permits the coverage fraction to be identified as a geometric probability, which can, in turn, be connected to a distribution of geometric scales of the set, as will be discussed below.

The size of the bounding box, $\delta_{b}$, provides a useful measure of the largest scale exhibited by the set. At this scale, the coverage count is unity (by construction), i.e.

$$
N_{d}\left(\delta_{b}\right)=1
$$

For the level sets considered above, for which the bounding box is the two-dimensional 
$(d=2)$ circumscribing rectangle, $\delta_{b}$ is given by the square-root of the area of the two-dimensional circumscribing rectangle; cf. (3.2). At all scales, the coverage count defined above has the property that

$$
\frac{\mathrm{d} N_{d}(\lambda)}{\mathrm{d} \lambda} \leqslant 0,
$$

i.e. at any scale smaller than the bounding-box scale, the coverage count will decrease (or remain constant) with decreasing scale (cf. Miller \& Dimotakis 1991a, Appendix).

The SDF (box) dimension is the scale-dependent generalization of the PLF dimension. It can be defined (e.g. Takayasu 1982, 1992; Miller \& Dimotakis 1991a; Dimotakis 1991), at a coverage scale $\lambda$, as

$$
D_{d}(\lambda) \equiv-\frac{\mathrm{d} \log N_{d}(\lambda)}{\mathrm{d} \log \lambda}
$$

in terms of the geometric coverage, $N_{d}(\lambda)$; cf. (5.1). The geometry of an object will be SDF if, in a range of scales,

$$
\frac{\mathrm{d} D_{d}(\lambda)}{\mathrm{d} \lambda} \neq 0
$$

while it will be PLF (i.e. $D_{d}=$ const.) if

$$
\frac{\mathrm{d} D_{d}(\lambda)}{\mathrm{d} \lambda}=0
$$

The SDF dimension, $D_{d}(\lambda)$, will tend to the topological dimension, $d_{t}$, at the smallest scales, and to the embedding dimension, $d$, at the largest scales, i.e.

$$
D_{d}(\lambda) \longrightarrow\left\{\begin{array}{l}
d_{t} \text { as } \lambda \rightarrow 0 \\
d \text { as } \lambda \rightarrow \delta
\end{array}\right.
$$

where $\delta$ is the largest characteristic scale of the set (cf. Dimotakis 1991). For spatial data confined in a bounding box, $\delta=\delta_{b}$, the bounding box size. If $D_{d}(\lambda)$ is monotonic with scale, these limiting values will also be the bounding values. SDF dimensions, defined through equations (5.4) and (5.5), allow for the description of phenomena whose geometric-scaling laws are scale-dependent, as can be expected to be admissible in general.

The SDF geometric-coverage law that follows from equation (5.4) is given, in differential form, by

$$
\frac{\mathrm{d} N_{d}(\lambda)}{N_{d}(\lambda)}=-D_{d}(\lambda) \frac{\mathrm{d} \lambda}{\lambda}
$$

i.e. the SDF dimension of a set, at a scale $\lambda$, can be identified as the fractional decrease in coverage, $-\mathrm{d} N_{d} / N_{d}$, per unit fractional increase in scale, $\mathrm{d} \lambda / \lambda$. Note that, if $D_{d}(\lambda) \neq$ const., the SDF differential-coverage relation (5.8) does not imply a power-law-like coverage, i.e. $N_{d}(\lambda) \not \subset \lambda^{-D_{d}(\lambda)}$; cf. (4.4).

Integrating the differential coverage relation (5.8), from a reference scale, $\lambda_{1}$, to a scale, $\lambda$, we see that a SDF dimension implies a geometric-scaling coverage law given by (cf. Takayasu 1982, 1992)

$$
\frac{N_{d}(\lambda)}{N_{d}\left(\lambda_{1}\right)}=\exp \left\{-\int_{\lambda_{1}}^{\lambda} D_{d}\left(\lambda^{\prime}\right) \frac{\mathrm{d} \lambda^{\prime}}{\lambda^{\prime}}\right\}=\exp \left\{\int_{\lambda}^{\lambda_{1}} D_{d}\left(\lambda^{\prime}\right) \frac{\mathrm{d} \lambda^{\prime}}{\lambda^{\prime}}\right\} .
$$


In particular, if the largest scale of the set, $\delta_{b}$, is the reference scale, we have

$$
N_{d}(\lambda)=\exp \left\{\int_{\lambda}^{\delta_{b}} D_{d}\left(\lambda^{\prime}\right) \frac{\mathrm{d} \lambda^{\prime}}{\lambda^{\prime}}\right\},
$$

since $N_{d}\left(\delta_{b}\right)=1$; cf. (5.2). Equation (5.10) should be contrasted with the scalelocal relation (4.4) for PLFs. For SDFs, the coverage can be seen to be a non-local function of scale, with geometric structure across the whole range of scales potentially contributing to the coverage at any one scale.

For the isoscalar contours considered above, the SDF coverage can be written, therefore, as

$$
N_{2}(\lambda)=\exp \left\{\int_{\lambda}^{\delta_{b}} D_{2}\left(\lambda^{\prime}\right) \frac{\mathrm{d} \lambda^{\prime}}{\lambda^{\prime}}\right\},
$$

where, for the two-dimensional space here, $\delta_{b}=\left(\lambda_{x} \lambda_{y}\right)^{\frac{1}{2}}$ is the size of the bounding box, with $\lambda_{x}$ and $\lambda_{y}$ the lengths of the two circumscribing-rectangle sides; $\mathrm{cf}$. (3.2).

A useful measure, related to the coverage, is the $d_{t}$-dimensional size of the set, defined as, $\lambda^{d_{t}} N_{d}(\lambda)$, where $d_{t}$ denotes the topological dimension of the set. In particular, for the level sets considered above $\left(d_{t}=d-1=1\right)$, this quantity becomes the coverage length of the isoscalar contours, $L_{2}(\lambda)$, cf. (4.2), and can be computed from

$$
\frac{L_{2}(\lambda)}{\delta_{b}} \equiv \frac{\lambda}{\delta_{b}} N_{2}(\lambda)
$$

where the coverage length is scaled by the size of the bounding box, $\delta_{b}$. The coverage length, at a scale $\lambda$, is, therefore, given by

$$
\frac{L_{2}(\lambda)}{\delta_{b}}=\frac{\lambda}{\delta_{b}} \exp \left\{\int_{\lambda}^{\delta_{b}} D_{2}\left(\lambda^{\prime}\right) \frac{\mathrm{d} \lambda^{\prime}}{\lambda^{\prime}}\right\} ;
$$

cf. (5.10). The small-scale limit of the coverage-length, i.e.

$$
\frac{L_{2}(\lambda \rightarrow 0)}{\delta_{b}}=\lim _{\lambda \rightarrow 0}\left[\frac{\lambda}{\delta_{b}} N_{2}(\lambda)\right],
$$

provides a finite measure, at the smallest scales, for one-dimensional $\left(d_{t}=1\right)$ level sets embedded in a two-dimensional space $(d=2)$, such as the isoscalar (level) sets considered above.

Another useful measure based on the coverage is the coverage fraction, $F_{d}(\lambda)$, or volume-fill fraction of the set at a scale $\lambda$, defined as (Dimotakis 1991)

$$
F_{d}(\lambda) \equiv \frac{N_{d}(\lambda)}{N_{d, t o t}(\lambda)}=\left(\frac{\lambda}{\delta_{b}}\right)^{d} N_{d}(\lambda)
$$

where $N_{d, t o t}(\lambda)$ is the total number of boxes of size $\lambda$ that can fit in the bounding box. For level sets derived from two-dimensional data $(d=2)$, the coverage fraction can be computed from

$$
F_{2}(\lambda) \equiv\left(\frac{\lambda}{\delta_{b}}\right)^{2} N_{2}(\lambda)
$$

cf. (5.12). The coverage fraction, as opposed to the SDF dimension, must be a non-decreasing function of scale, cf. (5.5), i.e.

$$
\frac{\mathrm{d} F_{d}(\lambda)}{\mathrm{d} \lambda} \geqslant 0
$$


at all scales. The logarithmic derivative of $F_{d}(\lambda)$ follows from equations (5.15) and (5.4), i.e.

$$
\frac{\mathrm{d} \log F_{d}(\lambda)}{\mathrm{d} \log \lambda}=d-D_{d}(\lambda)
$$

so that the behaviour of the coverage fraction is characterized by the embeddingdimension complement of the SDF dimension. The limiting behaviour of the coverage fraction is, cf. (5.7),

$$
F_{d}(\lambda) \sim \lambda^{d-d_{t}} \quad \text { as } \quad \lambda \rightarrow 0
$$

and

$$
F_{d}(\lambda) \rightarrow 1 \quad \text { as } \quad \lambda \rightarrow \delta_{b}
$$

Integrating equation (5.18) from a coverage scale, $\lambda$, to the largest scale, $\delta_{b}$, the SDF relation for the coverage fraction becomes, cf. (5.9),

$$
F_{d}(\lambda)=\exp \left\{-\int_{\lambda}^{\delta_{b}}\left[d-D_{d}\left(\lambda^{\prime}\right)\right] \frac{\mathrm{d} \lambda^{\prime}}{\lambda^{\prime}}\right\},
$$

since $F_{d}\left(\delta_{b}\right)=1$; cf. $(5.19 b)$. The degree to which a SDF set fills space, therefore, varies with scale and is dependent on the behaviour at other scales.

The implications of SDF geometry, as illustrated in equations (5.9), (5.13), and (5.20), are that geometric structures across a wide range of scales can contribute to the behaviour of various measures at any one scale, $\lambda$, such as the coverage, coverage length, or volume-fill fraction.

\section{Distribution of geometric scales}

The coverage statistics considered above can be related to a distribution of scales in the field of interest. Consider a set, $\mathscr{S}$, composed of various geometric structures, e.g. points, lines, surfaces, etc. The scalar-field level set, i.e. set of isoscalar contours embedded in the two-dimensional space, $E_{2}$, is such an example. The (box) coverage of $\mathscr{S}$ can be related to the distribution of (multidimensional) geometric scales spanned by $\mathscr{S}$, in the following sense.

Let $d$ be the (Euclidean) dimension of the embedding space $E_{d}$ (bounding box, e.g. circumscribing rectangle in two dimensions). For the two-dimensional level sets considered above, we have, $\delta_{b}=\left(\lambda_{x} \lambda_{y}\right)^{1 / 2}$. The coverage fraction, $F_{d}(\lambda)$, can be identified as the geometric probability that a (randomly placed) $\lambda$-box, interior to the (outer) $\delta_{b}$-box, covers part of $\mathscr{S}$; cf. (5.15). It is recognized here that the probability of covering the set with a $\lambda$-box, interior to the $\delta_{b}$-box, will, in general, be a function of position within the $\delta_{b}$-box. If the statistics are spatially inhomogeneous, as is the case for the isoscalar contours considered above, the function $F_{d}(\lambda)$ represents the probability of coverage for a $\lambda$-tile placed in the $\delta_{b}$-box without regard to its location.

The coverage fraction, $F_{d}(\lambda)$, is a cumulative distribution function of a measure of spatial scales. For a scale increment, $\Delta \lambda$, we have

$$
F_{d}(\lambda+\Delta \lambda) \equiv F_{d}(\lambda)+\int_{F_{d}(\lambda)}^{F_{d}(\lambda+\Delta \lambda)} \mathrm{d} F_{d}\left(\lambda^{\prime}\right) .
$$

The differential coverage fraction can be associated with measure of scales possessing its own probability density function, $f_{d}(\lambda)$, where

$$
f_{d}(\lambda) \equiv \frac{\mathrm{d} F_{d}(\lambda)}{\mathrm{d} \lambda}
$$


In this expression, $f_{d}(\lambda)$ is the probability density function of the largest empty-box scale, $\lambda$, i.e. the size of the largest box interior to the bounding $\delta_{b}$-box that is empty, i.e. covers no part of $\mathscr{S}$, as can be seen by the following considerations.

The identification of $f_{d}(\lambda)$, in (6.2), can be established by considering the probabilities of the following three events:

$\mathscr{A} \equiv\left\{(\lambda+\Delta \lambda)\right.$-box, in $\delta_{b}$-box, covers part of $\left.\mathscr{S}\right\}$,

$\mathscr{B} \equiv\left\{\lambda\right.$-box, in $\delta_{b}$-box, covers part of $\left.\mathscr{S}\right\}$,

$\mathscr{C} \equiv\left\{\Delta \lambda / 2\right.$-wide strip, around $\lambda$-box interior to $\delta_{b}$-box, covers part of $\left.\mathscr{S}\right\}$.

The geometric probabilities of these coverage events are related as follows:

$$
\mathscr{P}\{\mathscr{A}\}=\mathscr{P}\{\mathscr{B} \cup \mathscr{C}\} \equiv \mathscr{P}\{\mathscr{B}\}+\mathscr{P}\{\mathscr{C} \cap \overline{\mathscr{B}}\}
$$

Since

$$
F_{d}(\lambda+\Delta \lambda)=\mathscr{P}\{\mathscr{A}\} \quad \text { and } \quad F_{d}(\lambda)=\mathscr{P}\{\mathscr{B}\}
$$

we have, cf. (6.1),

$$
\int_{F_{d}(\lambda)}^{F_{d}(\lambda+\Delta \lambda)} \mathrm{d} F_{d}\left(\lambda^{\prime}\right)=\mathscr{P}\{\mathscr{C} \cap \overline{\mathscr{B}}\},
$$

which can be identified as the probability that the $\Delta \lambda / 2$-wide strip, around a $\lambda$-box interior to the $\delta_{b}$-box, covers part of $\mathscr{S}$ and that the $\lambda$-box is empty.

This allows the connection between the coverage statistics and the distribution of this (multidimensional) measure of spatial scales, $\lambda$. In this context, this scale is identified as the size of the largest empty box, inside the $\delta_{b}$-box, that contains a randomly located point, $P$, but contains no part of $\mathscr{S}$, i.e. is empty. Equivalently, the scale $\lambda$ is a measure of (twice) the distance from a point $P$ to the nearest element of $\mathscr{S}$.

From equations $(5.19 a, b)$ and $(6.2)$ we see that $f_{d}(\lambda)$ satisfies the required normalization condition over the range of spatial scales, i.e.

$$
\int_{0}^{\delta_{b}} f_{d}(\lambda) \mathrm{d} \lambda=F_{d}\left(\delta_{b}\right)-F_{d}(0)=1 .
$$

Integrating equation (6.2), we have the relation for the SDF coverage,

$$
F_{d}(\lambda)=\int_{0}^{\lambda} f_{d}\left(\lambda^{\prime}\right) \mathrm{d} \lambda^{\prime}
$$

The SDF dimension, $D_{d}(\lambda)$, can be expressed, therefore, in terms of the distribution of largest empty-box scales, $f_{d}(\lambda)$, cf. (5.18), i.e.

$$
D_{d}(\lambda)=d-\frac{\lambda f_{d}(\lambda)}{\int_{0}^{\lambda} f_{d}\left(\lambda^{\prime}\right) \mathrm{d} \lambda^{\prime}} .
$$

This can be inverted to yield the largest empty-box scale p.d.f. from the SDF dimension, $D_{d}(\lambda)$, directly, i.e.

$$
f_{d}(\lambda)=\frac{d-D_{d}(\lambda)}{\lambda} F_{d}(\lambda)=\frac{d-D_{d}(\lambda)}{\lambda} \exp \left\{-\int_{\lambda}^{\delta_{b}}\left[d-D_{d}\left(\lambda^{\prime}\right)\right] \frac{\mathrm{d} \lambda^{\prime}}{\lambda^{\prime}}\right\} ;
$$

cf. equations (5.20) and (6.2).

The small-scale behaviour of $f_{d}(\lambda)$ will be given by (cf. equations (5.19a) and (6.2))

$$
f_{d}(\lambda) \sim \lambda^{d-d_{t}-1} \rightarrow \begin{cases}\text { constant as } \lambda \rightarrow 0 & \text { for } d_{t}=d-1 \\ 0 \text { as } \lambda \rightarrow 0 & \text { for } d_{t}<d-1\end{cases}
$$


In particular, for the isoscalar contours considered above, for which $d_{t}=1$ and $d=2$, this can be related to the small-scale limit of the coverage length, cf. (5.13) and (5.14), i.e.

$$
\delta_{b} f_{2}(\lambda \rightarrow 0)=\frac{L_{2}(\lambda \rightarrow 0)}{\delta_{b}}
$$

The large-scale behaviour of $f_{d}(\lambda)$ will be given by, cf. $(5.19 a)$,

$$
f_{d}(\lambda) \rightarrow 0 \text { as } \lambda \rightarrow \delta_{b}
$$

Equivalently, the SDF dimension can be expressed in terms of the distribution of the logarithm of largest empty-box scales, $\tilde{f}_{d}(\log \lambda)$, i.e.

$$
D_{d}(\lambda)=d-\frac{\tilde{f}_{d}(\log \lambda)}{\int_{-\infty}^{\log \lambda} \tilde{f}_{d}\left(\log \lambda^{\prime}\right) \mathrm{d} \log \lambda^{\prime}},
$$

cf. (6.8), where

$$
\tilde{f}_{d}(\log \lambda) \mathrm{d} \log \lambda \equiv f_{d}(\lambda) \mathrm{d} \lambda \quad \text { or } \quad \tilde{f}_{d}(\log \lambda)=\lambda f_{d}(\lambda)
$$

with a small-scale behaviour given by

$$
\tilde{f}_{d}(\log \lambda) \sim \lambda^{d-d_{t}} \rightarrow 0 \quad \text { as } \lambda \rightarrow 0
$$

unconditionally; cf. (6.10).

We conclude that SDF-geometric statistics, such as the coverage fraction or dimension, are invertible and can be used to compute the distribution of geometric scales. Equations (6.2)-(6.9) provide the connections between the fractal geometry (SDF or PLF) and the distribution of largest empty-box scales of the set being covered, in the $d$-dimensional embedding space.

\section{Geometry of isoscalar surfaces}

An analysis of the geometry of the isoscalar surfaces (contours, in two dimensions) was performed on the scalar-field data in these experiments in terms of the following measures: the coverage, $N_{2}(\lambda)$, the coverage length, $L_{2}(\lambda)$, the SDF dimension, $D_{2}(\lambda)$, the coverage fraction, $F_{2}(\lambda)$, and the distribution of largest empty-box scales, $f_{2}(\lambda)$ and $\tilde{f}_{2}(\log \lambda)$. These were investigated as a function of scalar threshold for each of the three jet Reynolds numbers in these experiments. Isosurfaces used in the study of these measures were computed as the level sets of the scalar-image data, using a bilinear B-spline representation of the jet-fluid concentration $c(x, y)$-surface derived from each image. This method removes several difficulties of conventional pixelbased contour-identification methods and yields a representation for the isosurfaces that is well suited for the investigation of coverage-based measures. A new method to compute an estimate of the coverage of these isosurfaces was also developed that removes several shortcomings of conventional box-counting methods. These are discussed in the Appendix.

Figure 15(a) shows the ensemble-averaged two-dimensional coverage count, $N_{2}(\lambda)$, of isoscalar surfaces for $R e \simeq 9.0 \times 10^{3}$. Coverage counts are plotted for three thresholds $c_{1}, c_{2}$, and $c_{3}$ (cf. indicated values in figure 8), with lines of increasing solidity denoting increasing scalar threshold. The points joined by straight-line segments in figure $15(a)$ correspond to the coverage counts computed at the indicated $\lambda$-scales of the partitioned bounding box (cf. the Appendix). The spatial scale, $\lambda$, 

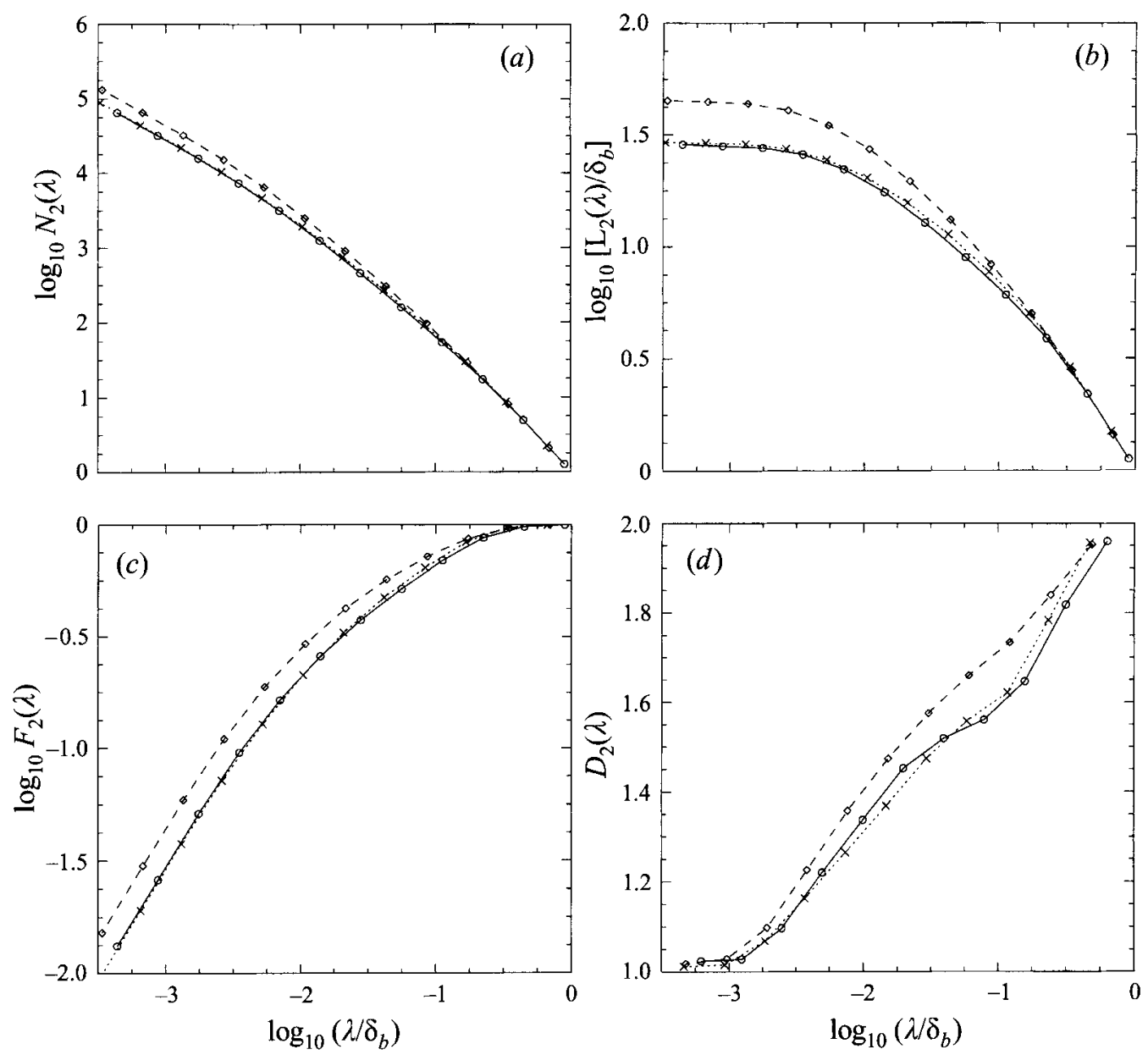

Figure 15. (a) Coverage, $N_{2}(\lambda),(b)$ normalized coverage length, $L_{2}(\lambda) / \delta_{b}(c),(c)$ coverage fraction, $F_{2}(\lambda),(d)$ SDF dimension, $D_{2}(\lambda)$, of isoscalar surfaces at $R e \simeq 9.0 \times 10^{3} . c=c_{1}$ : dotted line, crosses; $c=c_{2}$ : dashed line, diamonds; $c=c_{3}$ : solid line, circles. Recall (cf. figure 14) that $\delta_{b}=\delta_{b}(c ; R e)$.

is normalized by $\delta_{b}(c ; R e)$, the ensemble-averaged threshold-dependent boundingbox size (cf. figures 13,14, and related discussion). The coverage counts for the intermediate threshold, $c=c_{2}$, are seen to be larger than for $c=c_{1}$, or $c=c_{3}$, in accord with the scalar p.d.f. behaviour at this Reynolds number (cf. figure 8).

Figure $15(b)$ shows the ensemble-averaged coverage length, $L_{2}(\lambda)$, normalized by the bounding-box size, $\delta_{b}$, of isoscalar contours for $R e \simeq 9.0 \times 10^{3}$. The coverage length, $L_{2}(\lambda)$, defined in terms of the coverage in equation (5.12), will, in general, be different from (larger than) the arc-length of the (smooth) contours (cf. figure $18(b)$ in the Appendix). This geometric measure of the contours, with units of length, is seen to increase in a nonlinear fashion with decreasing scale, in logarithmic coordinates; cf. equations (4.1) and (5.13). The (normalized) coverage length is seen to be larger for the intermediate threshold, $c_{2}$, in accord with the p.d.f. behaviour of scalar values, at this Reynolds number (cf. figure 8). For the intermediate threshold, the small-scale limit of the coverage-length, cf. (5.14), is approximately equal to $L_{2}(\lambda \rightarrow 0) / \delta_{b} \simeq 45$ (cf. figure $15 b$ ), or normalized by the perimeter of a square $\delta_{b}$-box, 
$L_{2}(\lambda \rightarrow 0) /\left(4 \delta_{b}\right) \approx 11$. In other words, the turbulent-mixing process generates isoscalar contours with a small-scale coverage length, approximately 11 times longer than that of the perimeter of the bounding box at this scalar threshold and Reynolds number.

A plot of the coverage fraction, $F_{2}(\lambda)$, or volume-fill fraction (area-fraction in two dimensions), computed from the coverage counts of figure 15(a) using equation (5.16), is shown in figure $15(\mathrm{c})$. The coverage fraction is a normalized coverage count that is independent of the bounding-box size, $\delta_{b}(c ; R e)$. It can be seen to increase in a nonlinear fashion with increasing (logarithmic) scale; cf. equations (5.18) and (5.20). Its large-scale behaviour reflects the highest volume-fill fraction, with $F_{2}\left(\lambda \rightarrow \delta_{b}\right) \rightarrow 1$, as required. The asymptotic behaviour at the smallest scales corresponds to the lowest volume-fill fraction, i.e. $F_{2}(\lambda) \sim \lambda$, as $\lambda / \delta_{b} \rightarrow 0$, as expected for curves (onedimensional objects) in a two-dimensional space; cf. (5.19) and related discussion. The coverage fraction is largest at $c=c_{2}$, i.e. near the peak of the scalar p.d.f. (cf. figure 8).

The SDF dimension, $D_{2}(\lambda)$, derived from the data in figure 15(a), using equation $(5.4)(d)$, is shown in figure $15(d)$. The spatial scale, $\lambda$, is again normalized by the ensemble-averaged threshold-dependent bounding-box size, $\delta_{b}(c ; R e)$. The threshold dependence of the SDF dimension in figure $15(d)$ also reflects the scalar p.d.f. behaviour at this Reynolds number (cf. figure 8), with the SDF dimension largest, over most of the scale range, for the intermediate threshold, $c_{2}$. It is seen that $D_{2}(\lambda)$ is a function of scale and, in particular, not a constant. It is found to increase monotonically and continuously with scale, from near unity, at the smallest scales, to 2 , at the largest scales. In particular,

$$
\frac{\mathrm{d} D_{2}(\lambda)}{\mathrm{d} \lambda}>0
$$

at all scales spanned by these data, with

$$
d_{t}=1<D_{2}(\lambda)<2=d .
$$

The bounds are the topological dimension, $d_{t}=1$, and the embedding dimension, $d=2$, as expected for a monotonically increasing SDF dimension, $D_{2}(\lambda)$; cf. (5.7) and related discussion.

Figure 16(a) depicts the probability density function of largest empty-box scales, $f_{2}(\lambda)$, at $R e \simeq 9.0 \times 10^{3}$, for three scalar thresholds, computed using equation (6.2). This is a normalized probability density function, over the range of scales; cf. (6.6) and related discussion. For a given threshold, $f_{2}(\lambda)$ is seen to be larger at smaller scales, approaching a constant value at the smallest scales, as expected for level sets consisting of lines in a plane, i.e. for geometric sets with $d_{t}=d-1$; cf. (6.10) and related discussion. The data also indicate a higher probability density of largest emptybox scales, at small scales, for the $c_{2}$ threshold corresponding to the neighbourhood of the peak of the scalar p.d.f.

Perhaps more illustrative is the probability density function of the logarithm of largest empty-box scales, $\tilde{f}_{2}(\log \lambda)$. This is plotted in figure $16(b)$, for the same three scalar thresholds (cf. figure 8), at $R e \simeq 9.0 \times 10^{3}$. For a given threshold, $\tilde{f}_{2}(\log \lambda)$ approaches zero at the small as well as large scales, as expected; cf. equations (6.15) and (6.10). A higher probability density of largest empty-box scales is found at the smaller scales, for the $c_{2}$ threshold, in contrast to the behaviour at $c=c_{1}$ and $c=c_{3}$. This finding is discernible in the isosurface image data directly, as can be seen in figures $10(a)-10(c)$.

These statistics allow us to investigate the Reynolds number effects on the scalar field, isoscalar geometry, and scalar mixing. Figure 17(a) compares the ensemble- 

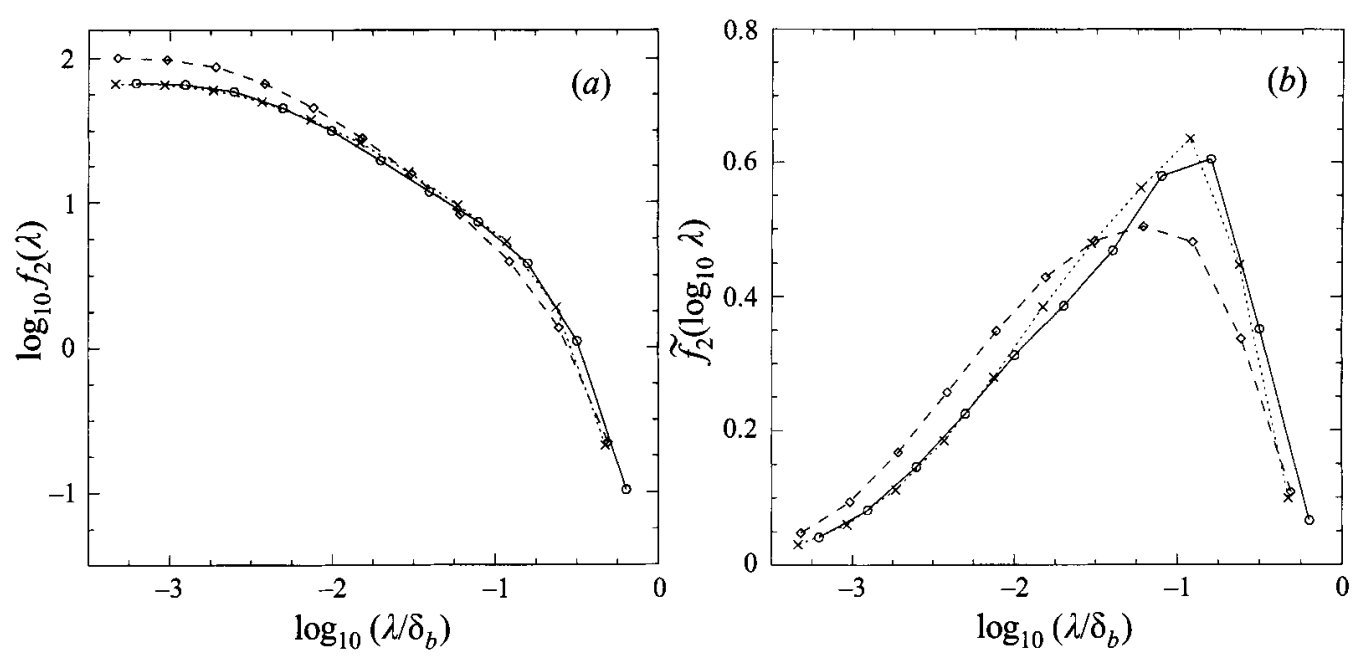

FIGURE 16. P.d.f. of $(a)$ largest empty-box scales, $f_{2}(\lambda),(b)$ the logarithm of largest empty-box scales, $\tilde{f}_{2}(\log \lambda)$, for isoscalar surfaces at $R e \simeq 9.0 \times 10^{3}$. Line/symbol legend as in figure 15 .

averaged coverage length, $L_{2}(\lambda)$, scaled by the isoscalar bounding-box size, $\delta_{b}$, at the three Reynolds numbers investigated, for the intermediate scalar threshold, $c_{2}$; cf. equation (5.12). Recall that, at this threshold and these Reynolds numbers, the bounding-box size, $\delta_{b}$, is approximately independent of Reynolds number (cf. figure 14 and related discussion). The data indicate that the scale-dependent coverage length, $L_{2}(\lambda)$, at a fixed scale, $\lambda$, decreases with increasing Reynolds number, at this scalar threshold. This, perhaps surprising, finding can be seen directly in the image data (cf. progression in figures $9 b, 10 b$, and $11 b$ ); it can be investigated further by comparing scale-local measures, such as the distribution of scales.

Figure 17(b) compares the isosurface SDF dimension at the three Reynolds numbers investigated, for the intermediate scalar threshold, $c_{2}$, with lines of increasing solidity denoting increasing Reynolds number. The data indicate that the SDF dimension decreases, in the range of moderate-to-large scales, as the Reynolds number increases, and at the same time, that at the smallest and largest scales, the SDF dimension is only weakly dependent on Reynolds number, if at all.

Figure $17(c)$ depicts the probability density function of the logarithm of largest empty-box scales, $\tilde{f}_{2}(\log \lambda)$, at the intermediate scalar threshold. A systematic $R e$ dependence is evident. In particular, at the smaller scales the data indicate a decreasing probability density of largest empty-box scales with increasing Reynolds number. In other words, at small scales it is less probable to find a $\lambda$-size region that is not visited by the isosurface, as the Reynolds number is increased.

\section{Conclusions}

Scalar-field measures and isoscalar measures derived from experimental investigations in the far field of liquid-phase turbulent jets are found to be functions of Reynolds number, with the latter also functions of scalar threshold. Analysis of these measures indicates a transition between different turbulent-mixing regimes, in the Reynolds number range $4.5 \times 10^{3} \leqslant R e \leqslant 18 \times 10^{3}$. The Reynolds-number dependence of such measures as the scalar p.d.f. (cf. figure 8), the area enclosed 

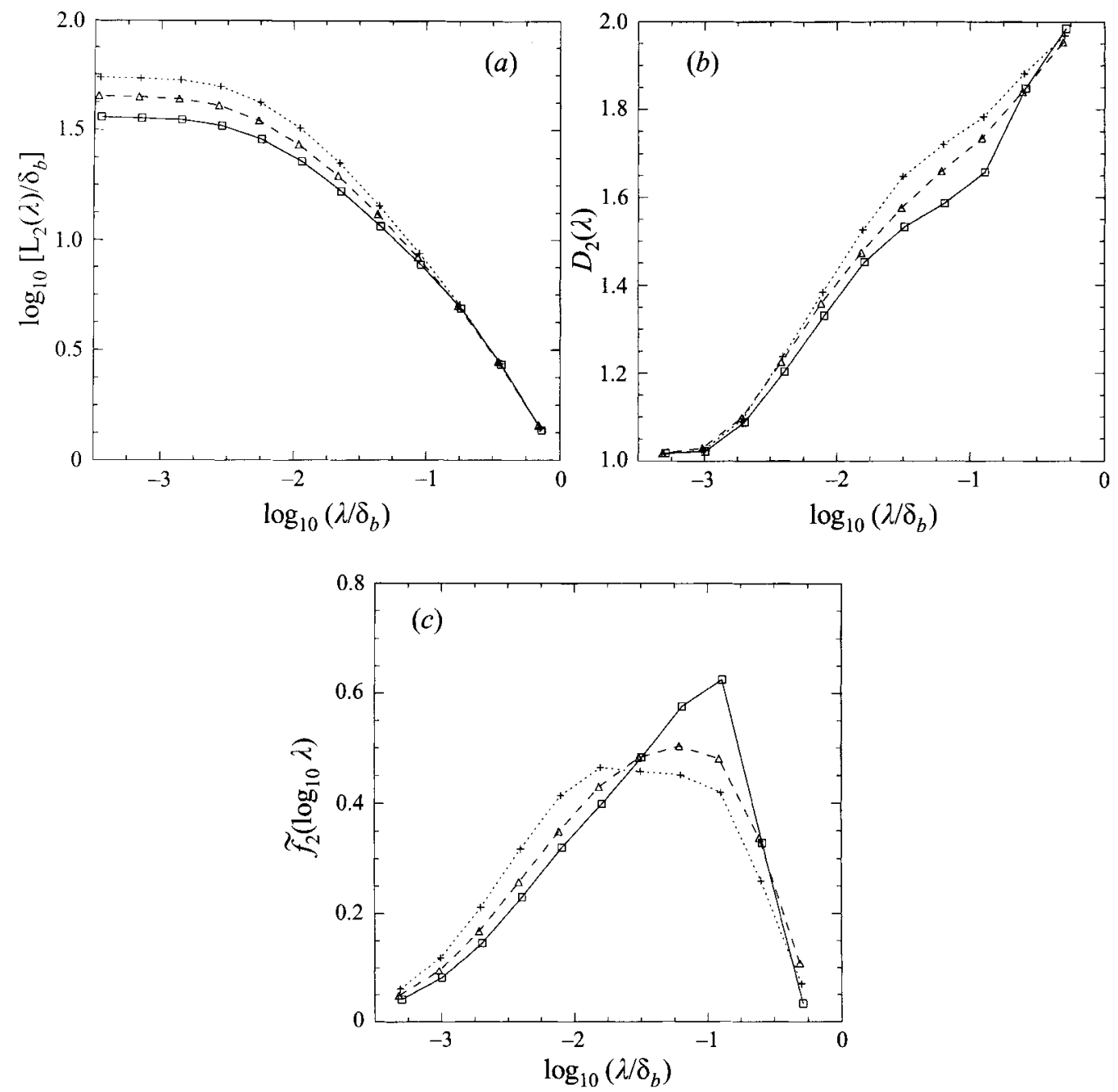

FIGURE 17. Reynolds-number dependence of $(a)$ coverage-length, $L_{2}(\lambda) / \delta_{b},(b)$ SDF dimension, $D_{2}(\lambda),(c)$ p.d.f. of the logarithm of largest empty-box scales, $\tilde{f}_{2}(\log \lambda)$, at the intermediate scalar threshold, $c=c_{2}$. $R e \simeq 4.5 \times 10^{3}:$ dotted line, crosses; $R e \simeq 9.0 \times 10^{3}:$ dashed line, triangles; $R e \simeq 18 \times 10^{3}:$ solid line, squares.

by the isosurfaces (cf. figure 12), and the spatial extent (bonding-box size) of the isosurfaces (cf. figure 14), is most manifest at lower scalar (jet-fluid concentration) values, corresponding to the outer region of the jet.

A conspicuous Reynolds-number dependence of measures derived from the coverage of scalar isosurfaces is found at the intermediate scalar threshold value, $c_{2}$, near the peak of the pre-transition scalar p.d.f.'s (cf. figure 8). For this threshold, the coverage length of the isoscalar contours decreases with increasing Reynolds number (cf. figure 17a). The SDF dimension is also found to decrease with increasing Reynolds number (cf. figure 17b), at the moderate-to-large scales, indicating an isosurface geometry that becomes less complex as the Reynolds number increases in this scale range. Consistent with these findings is the behaviour of the distribution of largest-empty-box scales, as the Reynolds number increases. In particular, the 
data indicate that small-scale regions of the flow are more likely to be visited by the isosurfaces: a lower probability of finding a largest-empty-box region of that size, as the Reynolds number is increased (cf. figure $17 \mathrm{c}$ ). Finally, the expectation value of the largest-empty-box scale (as well as the most probable) is increasing with increasing Reynolds number (cf. figure 17c). The distance from a point in the bounding box to the $c_{2}$ isosurface is increasing with increasing Reynolds number. These observations, taken collectively, indicate enhanced molecular mixing, that is responsible for (local) scalar-field homogenization, relative to stirring, that is responsible for isoscalar surface-area generation, with increasing Reynolds number. This occurs at thresholds corresponding to isoscalar surfaces (contours) mostly to be found in the intermediateradius (high-shear) regions of the jet (cf. $b$-series of figures 9-11). The imprint of this behaviour can also be seen in the dependence on Reynolds number of the highwavenumber portion of the spatial spectra; cf. figure 7. In particular, the scalar power spectra are seen to decrease with increasing Reynolds number at high wavenumbers. This observation is consistent with the notion of increased molecular mixing, resulting in a decrease in scalar variance with increasing Reynolds number. This was previously documented on the basis of (temporal) scalar fluctuation measurements on the centreline of liquid-phase turbulent jets (cf. Miller \& Dimotakis 1991b). Notably, this behaviour is not encountered in gas-phase jets, in the same Reynolds number range, and must therefore be attributed to the lower (liquid-phase) molecular diffusivity, i.e. Schmidt number effects (cf. Miller 1991, figure 7.2 and related discussion).

Conversely, the SDF dimension as well as the p.d.f. of largest-empty-box scales and other isoscalar statistics do not exhibit a discernible Reynolds number effect at low $\left(c=c_{1}\right)$ or high $\left(c=c_{3}\right)$ scalar values. Additionally, our data indicate that other scalar measures such as the area $A(c ; R e)$, cf. equation (3.1) and figure 12 , and bounding-box size $\delta_{b}(c ; R e)$, cf. figure 14 , do not possess a conspicuous Reynolds-number dependence at the intermediate threshold $\left(c=c_{2}\right)$.

The coverage of the isosurfaces is found to possess a fractal dimension that increases continuously with increasing scale, from near unity, at the smallest scales, to 2 , at the largest scales. The geometry of the isosurfaces is, therefore, found to be scaledependent fractal (SDF), and not power-law fractal (PLF), with a geometric complexity (here, fractal dimension) that increases monotonically with increasing scale.

The SDF properties expressed in equations (7.1) and (7.2) are probably generic. One can argue that they would also be encountered in other turbulent-mixing flows. From this general framework, one can expect that PLF geometry would be the exception, rather than the rule, i.e. that complex natural phenomena, in general, and turbulent flows, in particular, may be expected to exhibit SDF geometry.

This work was supported initially by AFOSR Grant 90-0304 and GRI Contract 5087-260-1467, and subsequently by AFOSR Grants F49620-92-J-0290 and F4962094-1-0353. It is part of a larger effort to investigate turbulent mixing in freeshear flows. The informal collaboration with P. Miller, at various stages of these investigations, the discussions with $\mathrm{C}$. Bond, as well as the contributions of D. Lang to the digital imaging in these experiments, are gratefully acknowledged.

\section{Appendix. Isosurface representation and coverage}

Conventional methods for the representation and coverage of isosurfaces (level sets) derived from digital images are based on the identification of boundary pixels. In the analysis of the data described here, the scalar image $c(x, y)$-surfaces were represented 
(a)

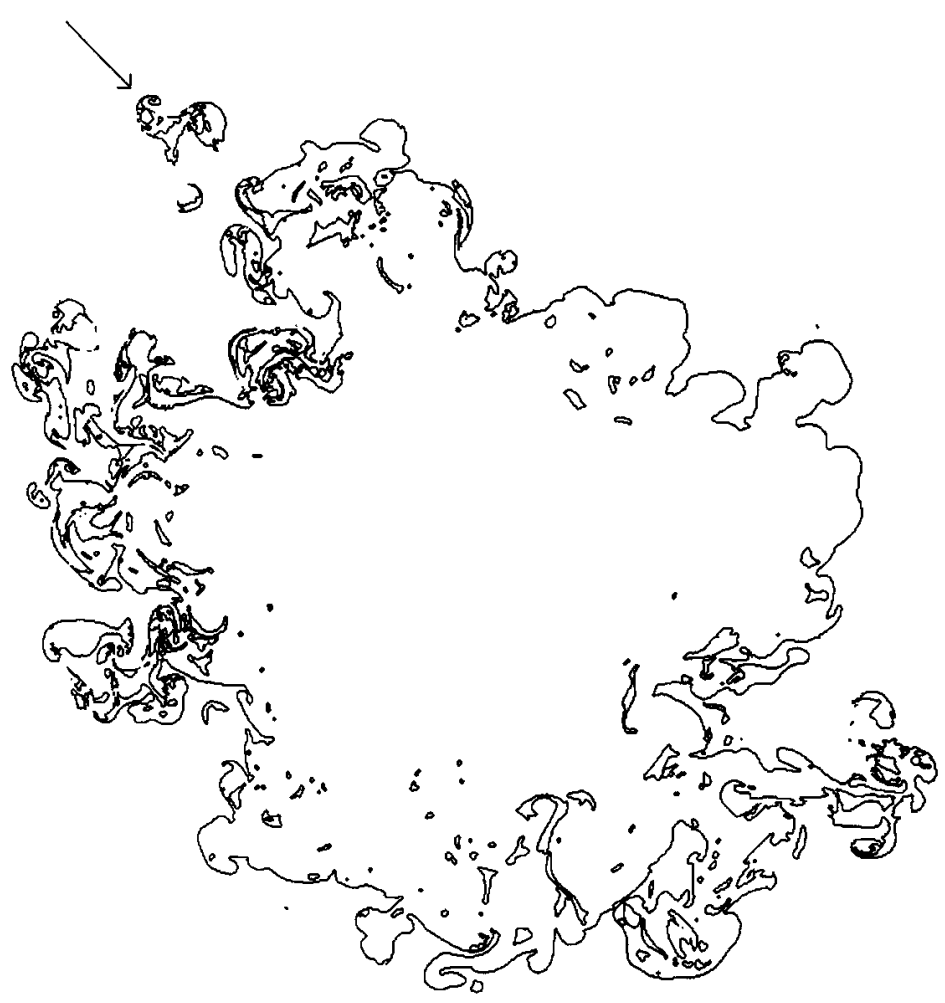

FIGURE 18(a). For caption see facing page.

using bilinear B-splines. Isoscalar contours (level sets) were then computed from the bilinear B-spline representation. This removes several difficulties associated with pixel-based schemes.

The effects of pixel representations on the identification of isosurfaces will be discussed first. Figure $18(a)$ depicts a scalar isosurface contour, for $c=c_{1}$, at $R e \simeq 9.0 \times 10^{3}$ (cf. figure 8) using conventional boundary pixels, within the field of view of the image. The field of view is, by design, larger than the local $\left(z / d_{0}=275\right)$ transverse jet extent. All individual level sets of the measured isosurfaces are thus closed and fully contained within the field of view.

A selected portion of the isosurface of figure 18(a), indicated by an arrow, is shown enlarged in figure $18(b)$ using conventional boundary pixels. The boundary-outlinepixel representation for this isosurface is also shown in figure $18(b)$, superimposed on the conventional-boundary-pixel representation. Boundary-outline pixels cover the outline of the boundary pixels and were devised and employed in the two- 


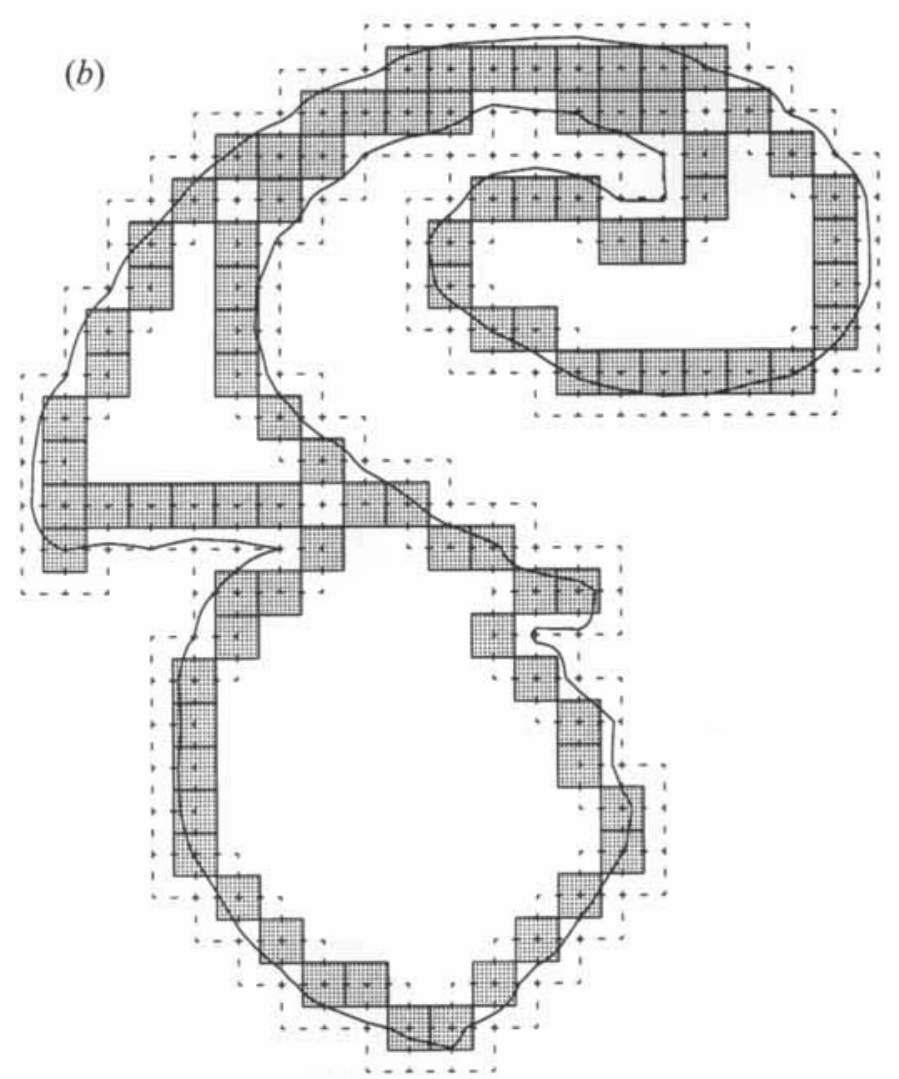

FIGURE 18. (a) Isoscalar surface at threshold $c=c_{1}$, for $R e \simeq 9.0 \times 10^{3}$ (cf. figure 8), depicted using conventional boundary pixels. Also shown is the field-of-view (dotted line). (b) Isoscalar island (indicated by arrow in $(a)$ ). Bilinear B-spline: solid line; boundary pixels: shaded squares; boundary-outline pixels: dashed squares.
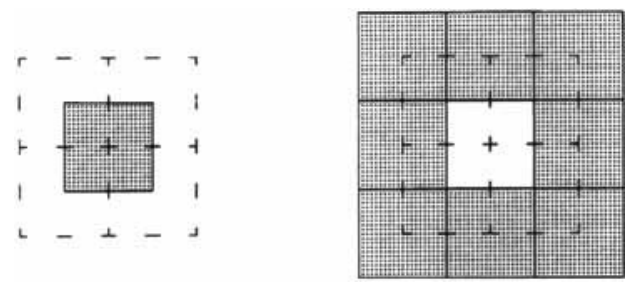

FIGURE 19. Asymmetry in conventional boundary-pixel representations. The boundary of a small island (left) and of a geometrically identical lake (right) is covered using conventional vs. boundary-outline pixels.

dimensional streak-image data analysis of Miller \& Dimotakis (1991a). Figure 19 shows a small island and a geometrically identical lake represented using both conventional and boundary-outline pixels. It is seen that the conventional-boundarypixel-representation counts for this island and lake are substantially different, even though the two isosurfaces are the same. There is, therefore, an inherent asymmetry in the representation of small lakes and islands using conventional boundary pixels. The boundary-outline-pixel representation removes this asymmetry.

Figure 20(a) compares the ensemble-averaged coverage counts of scalar isosurfaces 

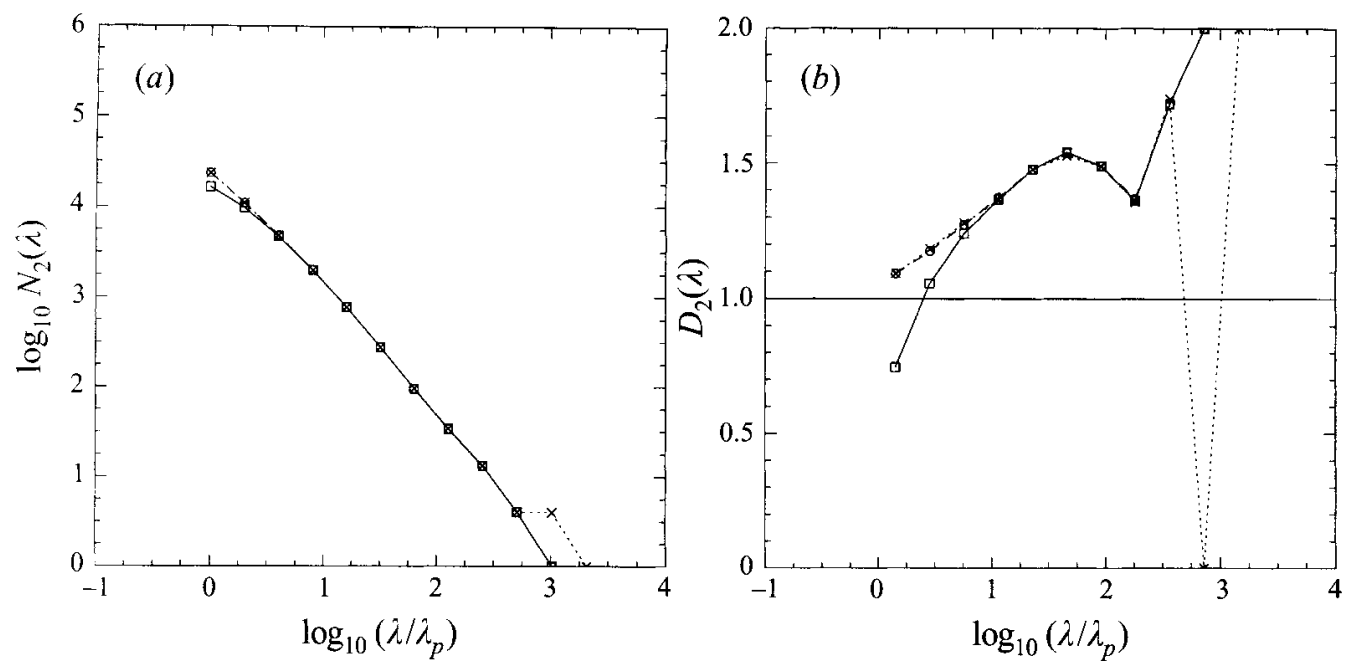

FiguRE 20. (a) Coverage, $N_{2}(\lambda)$, of scalar isosurfaces for $c=c_{1}$ at $R e \simeq 9.0 \times 10^{3}$ (cf. figure 8), computed by successive subdivision of the field of view (cf. figure 18a). Conventional boundary pixels: solid line, squares; boundary-outline pixels: dashed line, circles; larger field of view: dotted line, crosses. (b) Comparison of SDF dimension, $D_{2}(\lambda)$, computed for the coverage counts of $(a)$. The topological dimension, $d_{t}=1$, is also shown (solid line).

for $c=c_{1}$ at $R e \simeq 9.0 \times 10^{3}$ (cf. figure 8) represented using both conventional and boundary-outline pixels. The coverage counts for figure 20(a) were computed using a conventional coverage method in which the field of view of the image was successively subdivided (e.g. Sreenivasan et al. 1989). Figure 20(b) shows the corresponding ensemble-averaged SDF dimension, $D_{2}(\lambda)$. It is seen that the conventional-boundarypixel representation can lead to $D_{2}(\lambda)$ values at the smallest scales that are less than the topological dimension, $d_{t}$, i.e. unity, in this case. This is a result of the asymmetric representation of small lakes/islands with conventional-boundary pixels, as noted above. The boundary-outline-pixel representation removes this problem as shown in figure $20(b)$.

The coverage results of figure $20(a)$, however, exhibit an inflection point, at large scales, for both the conventional- and boundary-outline pixel representations. This is manifested as a dip in the value of $D_{2}(\lambda)$ at the large scales, as seen in the SDF dimension results in figure $20(b)$. This is an artifact of the fact that the conventional box-counting methods employed to produce the data in figure $20(b)$ subdivide the field of view of the image. Those methods do not account for the finite spatial extent of the data, and, as a result, such methods can mask the coverage behaviour of the data at the outer scales, producing counts that substantially overestimate the coverage counts at those scales. For example, the ensemble-averaged coverage counts based on successive subdivision of a larger field of view (2048 $\times 2048$ square-pixels) are also shown in figures $20(a)$ and $20(b)$. It is seen that a larger field of view, for the same data, can influence the large-scale estimate of the SDF dimension.

To address these issues, we have developed a modified box-counting method, the Bounding Box Partition method, that removes several shortcomings of conventional box-counting methods. The modified method accounts for the finite spatial extent of each particular isosurface, and is, therefore, able to produce coverage counts over the whole range of scales, from the image-pixel resolution to the spatial extent. The first step of the method is to estimate the spatial extent of each particular isosurface. 


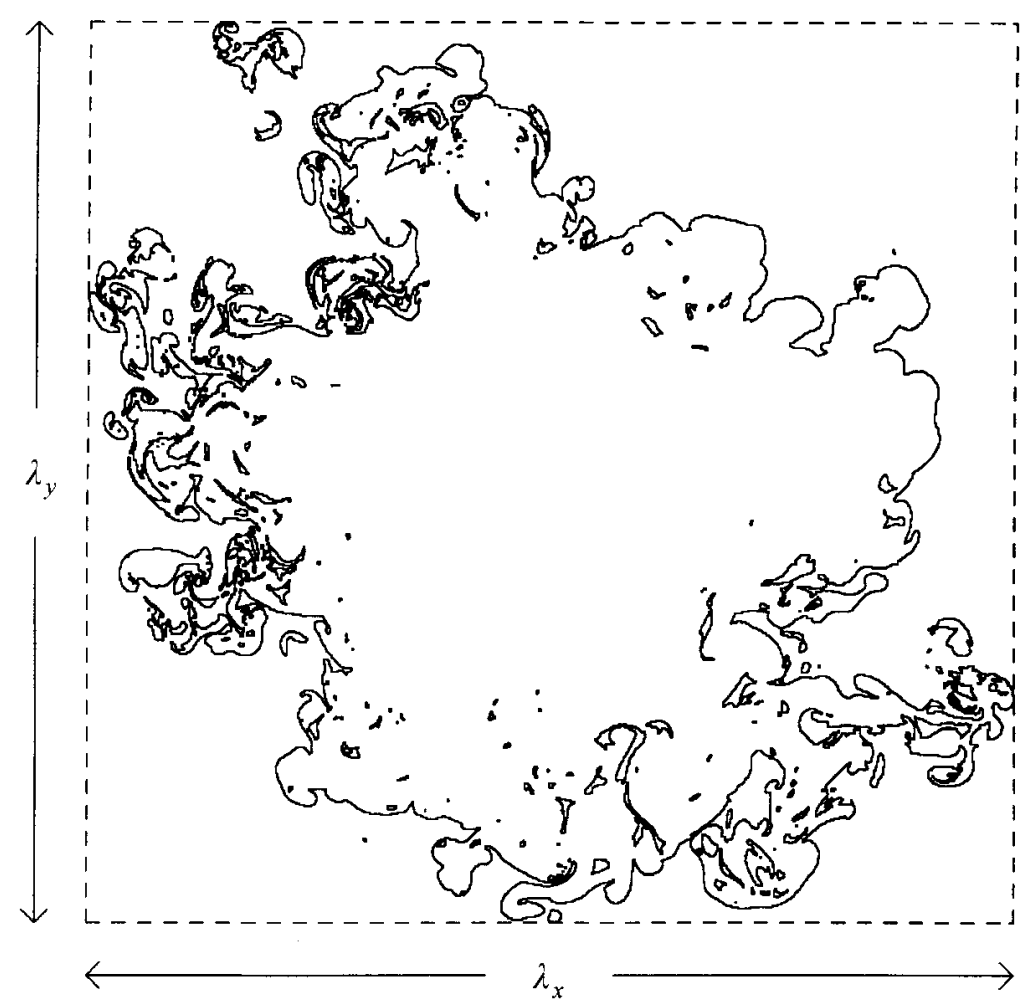

Figure 21. Bounding box (dashed line) computed for the isosurface of figure 18. Also shown is the field-of-view extent (dotted line). This isosurface is depicted using boundary-outline pixels.

This is achieved by identifying the bounding box, or smallest circumscribing rectangle (cf. Tricot 1995), that covers the isosurface. Figure 21 shows an example of a bounding box computed for the isosurface of figure 18(a), as well as the extent of the image field of view. A coverage count of unity is assigned for the bounding box. The single scale that corresponds to this coverage count is assigned to the geometric mean of the lengths of the two sides, $\lambda_{x}$ and $\lambda_{y}$, of the bounding box. This scale is also taken as an estimate of the spatial extent, $\delta_{b}$, of the isosurface, i.e.

$$
\delta_{b} \equiv\left(\lambda_{x} \lambda_{y}\right)^{1 / 2},
$$

and, cf. equation (5.2),

$$
N_{2}\left(\delta_{b}\right)=1
$$

The largest coverage scale, $\lambda^{(0)}$, at this initial stage of the coverage process, is given therefore by $\lambda^{(0)}=\delta_{b}$. In the first iteration, the bounding box is subdivided into four equal rectangles, and the number of the smaller rectangles covering parts of 

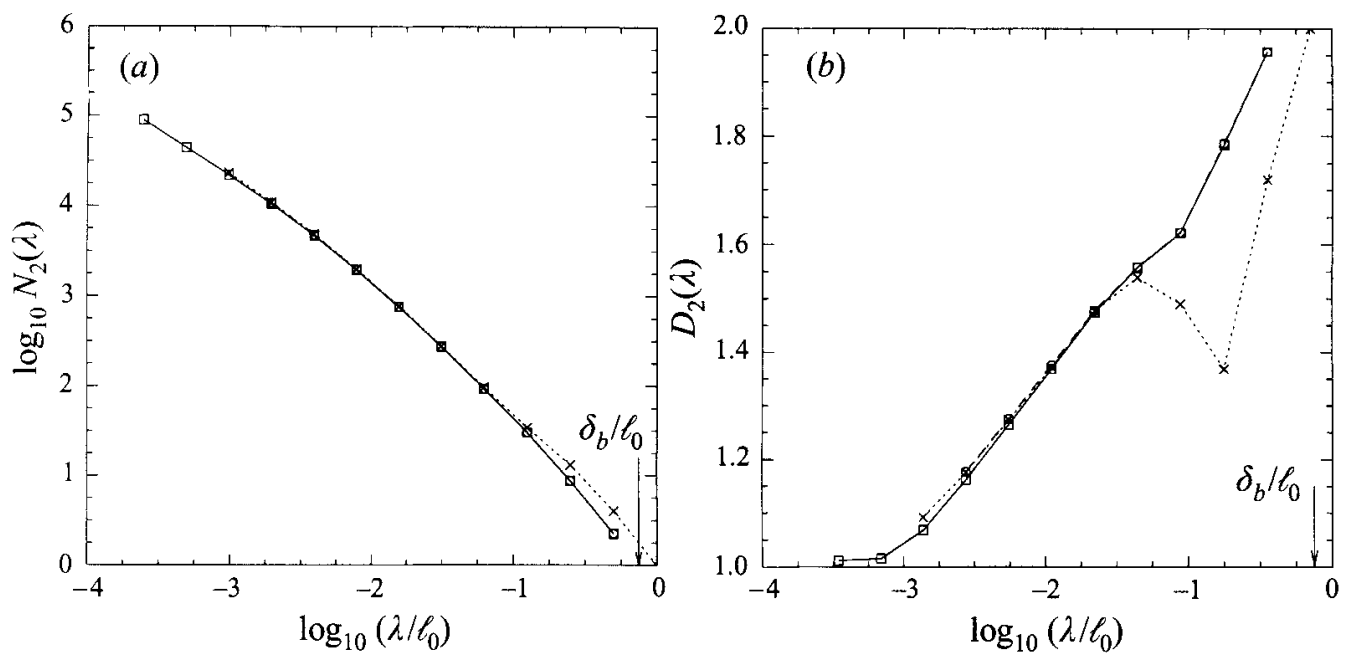

Figure 22. Coverage, $N_{2}(\lambda)$, of scalar isosurfaces for $c=c_{1}$ at $R e \simeq 9.0 \times 10^{3}$ (cf. figure 8), computed using the Rectangular Coverage Method. Bilinear B-splines: solid line, squares; boundary-outline pixels: dashed line, circles; Also shown is the field-of-view-based coverage (dotted line, crosses) (cf. figure 20(a)). (b) Comparison of SDF dimension, $D_{2}(\lambda)$, computed for the coverage counts of $(a)$

the isosurface is counted. The associated coverage scale, $\lambda^{(1)}$, corresponding to this (first-iteration) count is computed in a similar fashion, as the geometric mean of the sides of the smaller rectangles, so that $\lambda^{(1)}=\delta_{b} / 2$. The process is repeated by further subdividing each rectangle, so that, at the $k$ th iteration, the coverage scale is given by, $\lambda^{(k)}=\delta_{b} / 2^{k}$.

We now describe the bilinear B-spline representation method developed to represent the isosurfaces. This method removes the step-like pixelation difficulties that characterize pixel-based schemes. Bilinear B-splines conserve the (local) integral under the scalar surface, i.e. they match the particular pixel output. Figure 18(b) shows the bilinear B-spline representation (solid line) of an isosurface. For every set of four neighbouring image pixels, segments of the isosurfaces were computed using bilinear B-splines. Level sets of bilinear B-splines can, in general, give two branches within a four-pixel region, each of which belongs to a different isosurface. For this reason, a contour-following algorithm was written which marches along the boundary pixels belonging to the same isosurface. The resulting piecewise-bilinear isoscalar contours are continuous and closed (e.g. figure 18b).

The ensemble-averaged coverage computed using the Bounding Box Partition method applied to the bilinear B-spline isosurfaces is shown in figure 22(a), for scalar isosurfaces at $c=c_{1}$ and $R e \simeq 9.0 \times 10^{3}$. For comparison, figure 22(a) also shows the coverage counts computed using the Bounding Box Partition method for isosurfaces represented using boundary-outline pixels, as well as the coverage counts computed using the conventional field-of-view-based box-counting method applied to boundary-outline pixels. Figure 22(b) shows the ensemble-averaged SDF dimensions corresponding to the coverage counts of figure $22(a)$. It is seen that the Bounding Box Partition method removes the (artificial) dip in the value of $D_{2}(\lambda)$ at large scales associated with conventional box-counting methods.

The proposed Rectangular Coverage Method yields coverage counts at large scales that follow the actual spatial extent of the isosurfaces. Additionally, the coverage counts of the proposed method at small scales agree with the coverage counts obtained 
using the conventional method at those scales. Figures 22(a) and 22(b) also show that the bilinear B-spline representation yields coverage counts which agree, at the large scales, with the counts obtained using boundary-outline-pixel representations. At the small scales, boundary-outline-pixel representations lead to overestimates of the coverage necessary for the isosurfaces. Also, the smallest scale at which the conventional methods can produce coverage counts is limited by the pixel scale. The level sets derived from the bilinear B-spline representation, as seen in figure $22(b)$, lead to coverage results that do not have these limitations. The computed SDF dimension approaches unity at the smallest scales, as expected.

\section{REFERENCES}

Ashurst, W. T. 1995 A simple illustration of turbulent flame ball growth. Combust. Sci. Technol. 104, 19-32.

BiLger, R. W. 1980 Turbulent flows with nonpremixed reactants. In Turbulent Reacting Flows (ed. P. A. Libby \& F. A. Williams), pp. 65-113. Springer.

Borgas, M. S. 1993 The multifractal lagrangian nature of turbulence. Phil. Trans. R. Soc. Lond. A 342, 379-411.

Brandt, P. N., Greimel, R., Guenther, E. \& Mattig, W. 1991 Turbulence, fractals, and the solar granulation. In Applying Fractals in Astronomy (ed. A. Heck \& J. M. Perdang), pp. 77-96. Springer.

Castagnoli, C. \& Provenzale, A. 1991 From small-scale fractality to largescale homogeneity: a family of cascading models for the distribution of galaxies. Astro. Astrophys. 246, 634-643.

CHILÉS, J. P. 1988 Fractal and geostatistical methods for modelling of a fracture network. Math. Geol. 20, 631-654.

Constantin, P. 1990 Navier-Stokes equations and area of interfaces. Commun. Math. Phys. 129, 241-266.

Constantin, P. 1991 Remarks on the Navier-Stokes equations. In New Perspectives in Turbulence (ed. L. Sirovich), pp. 229-261. Springer.

Constantin, P. 1994a Geometric statistics in turbulence. SIAM Rev. 36, 73-98.

Constantin, P. $1994 b$ Geometric and analytic studies in turbulence. In Trends and Perspectives in Applied Mathematics (ed. L. Sirovich), pp. 21-54. Springer.

Constantin, P. \& Procaccia, I. 1994 The geometry of turbulent advection: sharp estimates for the dimensions of level sets. Nonlinearity 7, 1045-1054.

Constantin, P., Procaccia, I. \& Sreenivasan, K. R. 1991 Fractal geometry of isoscalar surfaces in turbulence: theory and experiment. Phys. Rev. Lett. 67, 1379-1742.

DimotaKis, P. E. 1991 Fractals, dimensional analysis and similarity, and turbulence. Nonlinear Sci. Today \#2/91, pp. 1, 27-31.

Dimotakis, P. E. 1993 Some issues on turbulent mixing and turbulence. GALCIT Rep. FM93-1a.

Dimotakis, P. E., Miake-Lye, R. C. \& Papantoniou, D. A. 1983 Structure and dynamics of round turbulent jets. Phys. Fluids 26, 3185-3192.

FALCONER, K. J. 1990 Fractal geometry: Mathematical foundations and applications. Wiley.

FloHR, P. \& Olivari, D. 1994 Fractal and multifractal characteristics of a scalar dispersed in a turbulent jet. Physica D 76, 278-290.

Gluckman, B. J., Willaime, H. \& Gollub, J. P. 1993 Geometry of isothermal and isoconcentration surfaces in thermal turbulence. Phys. Fluids 5, 647-661.

Hausdorff, F. 1919 Dimension und äusseres Mass. Math. Ann. 79, 157-179.

Kerstein, A. R. 1991 Linear-eddy modelling of turbulent transport. Part V: Geometry of scalar interfaces. Phys. Fluids A 3, 1110-1114.

Kolmogorov, A. N. \& Tiнomirov, V. M. $1959 \epsilon$-entropy and $\epsilon$-capacity of sets in functional spaces. Am. Math. Soc. Translations (1961, Section II), 277-364.

Kuznetsov, V. R. \& SABel'nikov, V. A. 1990 Turbulence and Combustion. Hemisphere.

LANE-SERFF, G. F. 1993 Investigation of the fractal structure of jets and plumes. J. Fluid Mech. 249, 521-534. 
Mandelbrot, B. B. 1967 How long is the coast of Britain? Statistical self-similarity and fractional dimension. Science 155, 636-638.

Mandelbrot, B. B. $1975 a$ On the geometry of homogeneous turbulence, with stress on the fractal dimension of the iso-surfaces of scalars. J. Fluid Mech. 72, 401-416.

Mandelbrot, B. B. $1975 b$ Les Objets Fractals: Forme, Hasard et Dimension. Flammarion.

Mandelbrot, B. B. 1977 Fractals, Form, Chance, and Dimension W. H. Freeman \& Co.

Mandelbrot, B. B. 1982 The Fractal Geometry of Nature W. H. Freeman \& Co.

Mandelbrot, B. B. 1989 Fractal geometry: What is it and what does it do? In Fractals in the Natural Sciences (ed. M. Fleischmann, D. J. Tildesley \& R. C. Ball), p. 7. Springer

MANTZARAS, J. 1992 Geometrical properties of turbulent premixed flames: comparison between computed and measured quantities. Combust. Sci. Tech. 86, 135-162.

Mark, D. M. \& Aronson, P. B. 1984 Scale-dependent fractal dimensions of topographic surfaces: an empirical investigation, with applications in geomorphology and computer mapping. Math. Geol. 16, 671-683.

MсСомв, W. D. 1991 The Physics of Fluid Turbulence Clarendon Press.

Mikhailov, A. S. \& Loskutov, A. Y. 1991 Foundations of Synergetics II: Complex Patterns Springer.

MiLler, P. L. 1991 Mixing in high Schmidt number turbulent jets. PhD thesis, California Institute of Technolgy.

Miller, P. L. \& Dimotakis, P. E. 1991a Stochastic geometric properties of scalar interfaces in turbulent jets. Phys. Fluids A3, 168-177.

Miller, P. L. \& Dimotakis, P. E. $1991 b$ Reynolds number dependence of scalar fluctuations in a high Schmidt number turbulent jet. Phys. Fluids A3, 1156-1163.

Prasad, R. R. \& SREenivasan, K. R. 1990 Quantitative three-dimensional imaging and the structure of passive scalar fields in fully turbulent flows. J. Fluid Mech. 216, 1-34.

Procaccia, I., Brandendurg, A., Jensen, M. H. \& Vincent, A. 1992 The fractal dimension of isovorticity structures in 3-dimensional turbulence. Europhys. Lett. 19, 183-187.

Richardoson, L. F. 1961 The problem of contiguity: an appendix of statistics of deadly quarrels. General Systems Yearbook 6, 139-187.

Rugaut, J.-P. 1991 Fractals, semi-fractals, and biometry. In Fractals: Non-integral Dimenstions and Applications (ed. G. Cherbit), pp. 151-187. Wiley.

Sreenivasan, K. R. 1991 Fractals and Multifractals in Fluid Turbulence. Ann. Rev. Fluid Mech. 23, 539-600.

Sreenivasan, K. R. 1994 Fractals in fluid mechanics. Fractals 2, 253-263.

Sreenivasan, K. R. \& Meneveau, C. 1986 The fractal facets of turbulence. J. Fluid Mech. 173, 357-386.

Sreenivasan, K. R., Prasad, R. R., Meneveau, C. \& Ramshankar, R. 1989 The fractal geometry of interfaces and the multifractal distribution of dissipation in fully turbulent flows. Pure Appl. Geoph. 131, 43-60.

SuzukI, M. 1984 Finite-size scaling for transient similarity and fractals. Prog. Theor. Phys. 71, $1397-1400$.

TAKayasu, H. 1982 Differential fractal dimension of random walk and its applications to physical systems. J. Phys. Soc. Japan 51, 3057-3064.

Takayasu, H. 1992 Fractals in the Physical Sciences Wiley.

TAYLoR, G. I. 1921 Diffusion by continuous movements. Proc. Lond. Math. Soc. 20, 196-212.

Tricot, C. 1995 Curves and Fractal Dimension Springer.

Vicsek, T. 1992 Fractal Growth Phenomena, 2nd Edn. World Scientific.

VINCENT, A. \& MENEGUZZI, M. 1991 The spatial structure and statistical properties of homogeneous turbulence. J. Fluid Mech. 225, 1-20.

WARE, B. R., Cyr, D., GoRTI, S. \& LANNI, F. 1983 Electrophoretic and frictional properties of particles in complex media measured by laser light scattering and fluorescence photobleaching rccovery. In Measurement of Suspended Particles by Quasi-Elastic Light Scattering, pp. 255-289. Wiley. 